\title{
Backreaction and Particle Production in (3+1)-dimensional QED
}

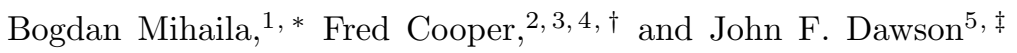 \\ ${ }^{1}$ Materials Science and Technology Division, Los Alamos National Laboratory, Los Alamos, NM 87545, USA \\ ${ }^{2}$ National Science Foundation, 4201 Wilson Blvd., Arlington, VA 22230, USA \\ ${ }^{3}$ Santa Fe Institute, Santa Fe, NM 87501, USA \\ ${ }^{4}$ Center for Nonlinear Studies, Los Alamos National Laboratory, Los Alamos, New Mexico 87545, USA \\ ${ }^{5}$ Department of Physics, University of New Hampshire, Durham, NH 03824, USA
}

\begin{abstract}
We study the fermion pair production from a strong electric field in boost-invariant coordinates in $(3+1)$ dimensions and exploit the cylindrical symmetry of the problem. This problem has been used previously as a toy model for populating the central-rapidity region of a heavy-ion collision (when we can replace the electric by a chromoelectric field). We derive and solve the renormalized equations for the dynamics of the mean electric field and current of the produced particles, when the field is taken to be a function only of the fluid proper time $\tau=\sqrt{t^{2}-z^{2}}$. We determine the propertime evolution of the comoving energy density and pressure of the ensuing plasma and the time evolution of suitable interpolating number operators. We find that unlike in $(1+1)$ dimensions, the energy density $\varepsilon$ closely follows the longitudinal pressure. The transverse momentum distribution of fermion pairs at large momentum is quite different and larger than that expected from the constant field result.
\end{abstract}

PACS numbers: 25.75.-q, 12.38.Mh

\section{INTRODUCTION}

The "Schwinger mechanism" for pair production has been used in various phenomenological models for particle production following a high-energy heavy-ion collision. One theoretical picture of high-energy heavy-ion collisions begins with the creation of a flux tube containing a strong color electric field [1]. The field energy is converted into particles such as $q \bar{q}$ pairs and gluons by the Schwinger mechanism 2, 3, 4. This mechanism has been implemented in a phenomenological fashion in event generators for particle production such as the Lund string model of hadronization [5] or the Hijing model [6]. More recently another picture of heavy-ion collisions, based on the color glass condensate model of high density for quantum chromodynamics (QCD) [7, 8] has been put forward. This model leads to the picture that a heavy-ion collision produces an initial semi-classical chromoelectric field in the longitudinal direction. Kharzeev et al. 9 have shown that if one looks at a perturbative parton cascade model and studies inclusive production of gluons in a gluon cascade, that this is equivalent to the production of a gluon from a background classical chromoelectric field in the longitudinal direction. This recent work gives credence to the idea that as far as gluon production is concerned, one can replace the dynamics of heavy-ion collisions by an initial condition on a semiclassical chromoelectric field. In these recent papers however, no attempt has been made to actually study the time evolution of the resulting plasma and the backreaction of the production on

\footnotetext{
*Electronic address: bmihaila@lanl.gov

†Electronic address: cooper@santafe.edu

$\ddagger$ Electronic address: john.dawson@unh.edu
}

the initial chromoelectric field. Some early studies had been done phenomenologically on this type of problem using a kinetic theory model in which a relativistic Boltzmann equation is coupled to a simple Schwinger source term 10, 11, 12, 13, and a Wigner function transport approach for an SU(2) version of QCD was recently done by Skokov and Levai [14. A first principle (quantum field theoretical) calculation for pair production and backreaction from strong fields was done by one of us and collaborators in the appropriate kinematics for heavy-ion collisions in $(1+1)$ dimensions in an abelian approximation where one ignored the color degrees of freedom. The reason for revisiting this problem now is two-fold. First, analytic results for the transverse momentum distribution functions for particles produced by constant electric and chromoelectric fields have recently been obtained [15, 16. For the constant chromoelectric field, the results for pair production are different than for an electric field in that the transverse distribution of jets depends not only on the energy density of the field but also on the direction the field is pointing in color space, i.e. the color hypercharge. Thus it is important to know first, how the backreaction affects the transverse momentum distribution function both for quantum electrodynamics (QED) and QCD and secondly whether adding interactions in a 2 -PI $1 / \mathrm{N}$ expansion will modify the one loop result. Here we will address the problem of finding the transverse distribution function for the abelian case in $(3+1)$ dimensions in a realistic kinematic scenario. The QCD problem will be addressed in a separate paper.

First let us review the history of both analytic approaches to the constant electric field and chromoelectric field problem as well as numerical studies of the backreaction problem. In his 1951 classic paper, Schwinger 
derived the following one-loop nonperturbative formula

$$
\frac{\mathrm{d} W}{\mathrm{~d}^{4} x}=\frac{e^{2} E^{2}}{4 \pi^{3}} \sum_{n=1}^{\infty} \frac{1}{n^{2}} e^{-n \pi m^{2} /|e E|}
$$

for the probability of fermion pair production per unit time per unit volume from a constant electric field $E$ via vacuum polarization [4] by using a proper time method. The result of Schwinger was extended to QCD by Claudson, Yildiz and Cox [17. However the $p_{T}$ distribution of the $e^{+}$(or $e^{-}$) production, $\mathrm{d} W / \mathrm{d}^{4} x \mathrm{~d}^{2} p_{T}$, could not be obtained using the proper time method of Schwinger. A WKB approximate method was used for this purpose by Casher et al. [18, but an exact method to do this problem (of determining the transverse distribution of pairs) was not found until recently [15, 16. For QED the WKB analysis gave the correct answer which depended only on the energy density of the electric field. However, for QCD, the WKB answer was incorrect for QCD in that it did not contain the second Casimir invariant of $S U(3)$, $C_{2}=\left[d_{a b c} E^{a} E^{b} E^{c}\right]^{2}$, as shown in Refs. 15] and 16, In the case of fermions in QED one finds for the transverse distribution of fermion pairs:

$$
\frac{\mathrm{d} W}{\mathrm{~d}^{4} x \mathrm{~d}^{2} p_{T}}=-\frac{|e E|}{4 \pi^{3}} \ln \left[1-e^{-\pi\left(p_{T}^{2}+m^{2}\right) /|e E|}\right] .
$$

The purpose of this paper is to consider the backreaction problem in $(3+1)$ dimensions in a situation which is related to the kinematics of particle production by strong chromoelectric fields, namely initial conditions where the center-of-mass energy is so high that all distribution functions are boost invariant in the longitudinal direction so that physical quantities only depend on the longitudinal proper time which is the same in the boost-invariant limit to the (1+1)-dimensional fluid proper time. Our goal is to see how the original result of Casher et al. [18, which has been recently rigorously derived by Nayak for the transverse distribution of fermion pairs [19, is modified by the expansion of the ensuing plasma and the backreaction on the electric field.

The backreaction problem was first studied numerically in real time for both scalar QED and QED by Cooper, Mottola and collaborators in $(1+1)$ dimensions [20, 21, 22] and then also in boost invariant coordinates relevant to heavy-ion collisions in Ref. 23. In Ref. [23], a strong abelian field was used as a model for particle production in the central-rapidity distribution. In that work, the boost invariance of the problem was used to show that many features of the hydrodynamical model were appearing even though there were no interactions kept that would lead to equilibration. Also, in that paper, although the theory was formulated in $(3+1)$ dimensions, numerical results were only presented for $(1+1)$ dimensions, so that the transverse distribution of secondaries was not studied. To remedy this particular deficiency of our previous work, here we investigate the dynamics of the particle production as a function of time in cylindrically-symmetric boost-invariant coordinates in $(3+1)$-dimensional QED.

The present paper builds on our previous papers on fermion pair production in $(1+1)$ dimensions by strong electric fields with a backreaction of the current on the field [23, 24]. As in our previous work, we employ quantum field theory methods in the large-N approximation to find the particle production rate. The next logical steps are to extend this result to QCD in this one-loop approximation and then to do a self-consistent resummed $1 / \mathrm{N}$ expansion to study the competition between the thermalization of the plasma and the expansion. In this way we will build up gradually the machinery to ask important questions about the thermalization and expansion of the quark-gluon plasma in a model based on the Schwinger mechanism.

The paper is organized as follows: In Sec. II we derive the equations needed for this calculation. In Sec. III we derive the components of the energy-momentum tensor in this coordinate system and show that it is conserved. In Sec. IV] we introduce the concept of the quasiparticle phase space distribution function. This quantity can be extracted from the field theory energy density and then used to determine the distributions of pairs produced in the center-of-mass frame. In Sec. $\mathrm{V}$ we discuss our numerical approach and present results of our calculations. We conclude in Sec. VI In App. A we explain the notation we use throughout this paper. In App. B we derive the transverse helicity eigenvectors we use in the main text of the paper to expand the fermi field, whereas in App. C we derive an adiabatic expansion of the Dirac equation which are used throughout the paper to study the large momentum behavior of integrands.

\section{THEORY AND NOTATION}

In cartesian coordinates $\xi^{a}=(t, x, y, z)$, and using the matric $\eta_{a b}=\operatorname{diag}(1,-1,-1,-1)$, the lagrangian density for this problem is given by

$$
\begin{aligned}
\mathcal{L}=\hat{\bar{\psi}}(\xi)\left\{\gamma^{a}\left[i \partial_{a}-e A_{a}(\xi)\right]-M\right\} \hat{\psi}(\xi) & -\frac{1}{4} F^{a b}(\xi) F_{a b}(\xi),
\end{aligned}
$$

where $F^{a b}(\xi)=\partial^{a} A^{b}(\xi)-\partial^{b} A^{a}(\xi)$. Equations of motion are given by

$$
\begin{array}{r}
\left\{\gamma^{a}\left[i \partial_{a}-g A_{a}(\xi)\right]-M\right\} \hat{\psi}(\xi)=0, \\
\partial_{a} F^{a b}(\xi)=\left\langle\hat{j}^{b}(\xi)\right\rangle=g\left\langle\left[\hat{\bar{\psi}}(\xi), \gamma^{b} \hat{\psi}(\xi)\right]\right\rangle / 2 .
\end{array}
$$

In this semiclassical approximation, we quantize the Dirac field while the electromagnetic field is treated classically. This approximation can be made precise by considering $N$ flavors of quarks interacting with the electromagnetic field and considering the limit where $N \rightarrow \infty$ after appropriate re-scalings. Systematic corrections are given by the $1 / N$ expansion as discussed in Ref. 25 and 
references therein. We use the standard representation of the $\gamma$-matrices

$$
\gamma^{0}=\left(\begin{array}{cc}
1 & 0 \\
0 & -1
\end{array}\right), \quad \gamma^{i}=\left(\begin{array}{cc}
0 & \sigma^{i} \\
-\sigma^{i} & 0
\end{array}\right)
$$

where $\sigma^{i}$ are the usual Pauli matrices, and 1 is the unit $(2 \times 2)$ matrix.

\section{A. Dirac's equation in boost-invariant coordinates}

Boost-invariant coordinates $x^{\mu}=(\tau, \rho, \theta, \eta)$ are defined by

$$
\begin{array}{ll}
t=\tau \cosh \eta, & z=\tau \sinh \eta, \\
x=\rho \cos \theta, & y=\rho \sin \theta .
\end{array}
$$

We use Roman indices to indicate the cartesian frame and Greek indices for the cylindrical-hyperbolic frame. The connection between the cartesian frame $\left(\mathrm{d} \xi^{a}\right)$, and the boost-invariant frame $\left(\mathrm{d} x^{\mu}\right)$ is described by a vierbein matrix $V^{a}{ }_{\mu}(x)$, which for our case is

$$
\mathrm{d} \xi^{a}=V_{\mu}^{a}(x) \mathrm{d} x^{\mu}, \quad \partial_{\mu}=V_{\mu}^{a}(x) \partial_{a},
$$

with

$$
V_{\mu}^{a}(x) \equiv \frac{\partial \xi^{a}}{\partial x^{\mu}}=\left(\begin{array}{cccc}
\cosh \eta & 0 & 0 & \tau \sinh \eta \\
0 & \cos \theta & -\rho \sin \theta & 0 \\
0 & \sin \theta & \rho \cos \theta & 0 \\
\sinh \eta & 0 & 0 & \tau \cosh \eta
\end{array}\right)
$$

The inverse vierbein matrix, which we write as $V^{\mu}{ }_{a}(x)$, is given by

$$
\mathrm{d} x^{\mu}=V^{\mu}{ }_{a}(x) \mathrm{d} \xi^{a}, \quad \partial_{a}=V^{\mu}{ }_{a}(x) \partial_{\mu},
$$

with

$$
\begin{aligned}
& V_{a}^{\mu}(x) \equiv \frac{\partial x^{\mu}}{\partial \xi^{a}} \\
& =\left(\begin{array}{cccc}
\cosh \eta & 0 & 0 & -\sinh \eta \\
0 & \cos \theta & \sin \theta & 0 \\
0 & -\sin \theta / \rho & \cos \theta / \rho & 0 \\
-\sinh \eta / \tau & 0 & 0 & \cosh \eta / \tau
\end{array}\right) .
\end{aligned}
$$

The metric $g_{\mu \nu}(x)$ in boost-invariant coordinates is

$$
\begin{aligned}
g_{\mu \nu}(x) & =\eta_{a b} V_{\mu}^{a}(x) V^{b}{ }_{\nu}(x) \\
& =\operatorname{diag}\left(1,-1,-\rho^{2},-\tau^{2}\right) .
\end{aligned}
$$

We raise and lower Latin indices by the $\eta$-metric and Greek indices by the $g$-metric. So Dirac's equation in boost-invariant coordinates can be written as

$$
\left\{\tilde{\gamma}^{\mu}(x)\left[i \partial_{\mu}-e A_{\mu}(x)\right]-M\right\} \hat{\psi}(x)=0,
$$

where we have defined $\tilde{\gamma}^{\mu}(x)=\gamma^{a} V^{\mu}{ }_{a}(x)$. In this coordinate system, the $\gamma$-matrices are given by

$$
\begin{aligned}
& \tilde{\gamma}^{\tau}(x)=\cosh \eta \gamma^{0}-\sinh \eta \gamma^{3}, \\
& \tilde{\gamma}^{\rho}(x)=\cos \theta \gamma^{1}+\sin \theta \gamma^{2}, \\
& \tilde{\gamma}^{\theta}(x)=\left(-\sin \theta \gamma^{1}+\cos \theta \gamma^{2}\right) / \rho, \\
& \tilde{\gamma}^{\eta}(x)=\left(-\sinh \eta \gamma^{0}+\cosh \eta \gamma^{3}\right) / \tau .
\end{aligned}
$$

The fermi field $\hat{\psi}(x)$ in boost-invariant coordinates obeys the anticommutation relation

$$
\begin{aligned}
& \left\{\hat{\psi}_{\alpha}(\tau, \rho, \theta, \eta), \hat{\bar{\psi}}_{\alpha^{\prime}}^{\dagger}\left(\tau, \rho^{\prime}, \theta^{\prime}, \eta^{\prime}\right)\right\} \\
& \quad=\tilde{\gamma}_{\alpha, \alpha^{\prime}}^{\tau}(\eta) \frac{\delta\left(\rho-\rho^{\prime}\right)}{\sqrt{\rho \rho^{\prime}}} \delta\left(\theta-\theta^{\prime}\right) \frac{\delta\left(\eta-\eta^{\prime}\right)}{\tau} .
\end{aligned}
$$

It is simpler, however, to solve Dirac's equation in a Lorentz-transformed frame which diagonalizes the vierbein. The Lorentz transformation that does this is

$$
\begin{aligned}
\Lambda_{b}^{a}(\theta, \eta) & =V_{\mu}^{a}(\tau, \rho, \theta, \eta) \bar{V}_{b}^{\mu}(\tau, \rho) \\
& =\left(\begin{array}{cccc}
\cosh \eta & 0 & 0 & \sinh \eta \\
0 & \cos \theta & -\sin \theta & 0 \\
0 & \sin \theta & \cos \theta & 0 \\
\sinh \eta & 0 & 0 & \cosh \eta
\end{array}\right),
\end{aligned}
$$

where $\bar{V}^{\mu}{ }_{b}(\tau, \rho)$ are the diagonal vierbeins given by

$$
\begin{aligned}
& \bar{V}^{a}{ }_{\mu}(\tau, \rho) \equiv V^{a}{ }_{\mu}(\tau, \rho, 0,0)=\operatorname{diag}(1,1, \rho, \tau) \\
& \bar{V}^{\mu}{ }_{a}(\tau, \rho) \equiv V^{\mu}{ }_{a}(\tau, \rho, 0,0)=\operatorname{diag}(1,1,1 / \rho, 1 / \tau) .
\end{aligned}
$$

We define $\gamma$-matrices in this frame with a bar

$$
\bar{\gamma}^{\mu}(\tau, \rho)=\bar{V}_{a}^{\mu}(\tau, \rho) \gamma^{a}=\tilde{\gamma}^{\mu}(\tau, \rho, 0,0)
$$

They are given explicitly by:

$$
\begin{array}{ll}
\bar{\gamma}^{\tau}=\gamma^{0}, & \bar{\gamma}^{\eta}(\tau)=\gamma^{3} / \tau, \\
\bar{\gamma}^{\rho}=\gamma^{1}, & \bar{\gamma}^{\theta}(\rho)=\gamma^{2} / \rho .
\end{array}
$$

Now let $S(\theta, \eta)$ be an operator which induces this Lorentz transformation on the $\gamma^{a}$ matrices in the orthogonal frame,

$$
S^{-1}(\theta, \eta) \gamma^{a} S(\theta, \eta)=\Lambda_{b}^{a}(\theta, \eta) \gamma^{b}
$$

Then it is easy to show that $S(\theta, \eta)=S_{\rho}(\theta) S_{\rho}(\eta)$ is given by a product of operators, where

$$
\begin{aligned}
S_{\rho}(\theta) & =\exp \left[\theta \gamma^{1} \gamma^{2} / 2\right] \\
& =\cos (\theta / 2)+\gamma^{1} \gamma^{2} \sin (\theta / 2) \\
S_{\tau}(\eta) & =\exp \left[\eta \gamma^{0} \gamma^{3} / 2\right] \\
& =\cosh (\eta / 2)+\gamma^{0} \gamma^{3} \sinh (\eta / 2)
\end{aligned}
$$

Furthermore, from Eq. 2.13, we see that $S(\theta, \eta)$ transforms the $\tilde{\gamma}^{\mu}(\tau, \rho, \theta, \eta)$ matrices into the $\bar{\gamma}^{\mu}(\tau, \rho)$,

$$
S^{-1}(\theta, \eta) \tilde{\gamma}^{\mu}(x) S(\theta, \eta)=\bar{\gamma}^{\mu}(\tau, \rho) \text {. }
$$


Now let us note that

$$
\begin{aligned}
& S^{-1}(\theta, \eta) \tilde{\gamma}^{\mu}(x) \partial_{\mu} S(\theta, \eta) \\
& =\bar{\gamma}^{\mu}(\tau, \rho)\left[\partial_{\mu}+\Pi_{\mu}(\theta, \eta)\right],
\end{aligned}
$$

where we have defined a connection $\Pi_{\mu}(\theta, \eta)$ by

$$
\Pi_{\mu}(\theta, \eta)=S^{-1}(\theta, \eta)\left(\partial_{\mu} S(\theta, \eta)\right) .
$$

The only nonzero connections are when $\mu=\theta$ and $\mu=\eta$. So using Eq. 2.18), we find

$$
\Pi_{\theta}=\gamma^{1} \gamma^{2} / 2, \quad \text { and } \quad \Pi_{\eta}=\gamma^{0} \gamma^{3} / 2,
$$

which are independent of $\theta$ or $\eta$. The covariant derivative $\nabla_{\mu}$ is given by

$$
\nabla_{\mu} \equiv D_{\mu}+\Pi_{\mu}(x) \equiv \partial_{\mu}+\Pi_{\mu}(x)+i e A_{\mu}(x) .
$$

Christoffel symbols for boost-invariant coordinates, which we will need later, are given by

$$
\Gamma_{\mu \nu}^{\lambda}(x)=V^{\lambda}{ }_{a}(x) \partial_{\mu} V^{a}{ }_{\nu}(x),
$$

from which we find the only non-vanishing elements to be

$$
\begin{array}{cc}
\Gamma_{\eta \eta}^{\tau}=\tau, & \Gamma_{\tau \eta}^{\eta}=\Gamma_{\eta \tau}^{\eta}=1 / \tau, \\
\Gamma_{\theta \theta}^{\rho}=-\rho, & \Gamma_{\rho \theta}^{\theta}=\Gamma_{\theta \rho}^{\theta}=1 / \rho .
\end{array}
$$

So Dirac's equation $(2.10)$ can be transformed to the boost-invariant frame by defining

$$
\hat{\psi}(x)=S(\theta, \eta) \hat{\phi}(x) / \sqrt{\tau},
$$

and multiplying the equation through by $S^{-1}(\theta, \eta)$, which gives the equation

$$
\left[i \bar{\gamma}^{\mu}(\tau, \rho) \nabla_{\mu}-M\right] \hat{\phi}(x) / \sqrt{\tau}=0 .
$$

For our case, we assume that the vector potential is in the $\eta$-direction and depends only on $\tau$, so we choose $A_{\mu}(x)=$ ( $0,0,0,-A(\tau))$, which defines $A(\tau)$ as the negative of the covariant component. Then (2.27) simplifies to

$$
\begin{aligned}
& \left\{i \gamma^{0} \partial_{\tau}+i \gamma^{1}\left[\partial_{\rho}+1 /(2 \rho)\right]+i \gamma^{2} \partial_{\theta} / \rho\right. \\
& \left.\quad+\gamma^{3}\left[i \partial_{\eta}+e A(\tau)\right] / \tau-M\right\} \hat{\phi}(x)=0,
\end{aligned}
$$

which is the equation we want to solve. Here $\hat{\phi}(x)$ field obeys the simpler anticommutation relation

$$
\left\{\hat{\phi}_{\alpha}(\tau, \mathbf{x}), \hat{\phi}_{\alpha^{\prime}}^{\dagger}\left(\tau, \mathbf{x}^{\prime}\right)\right\}=\delta_{\alpha, \alpha^{\prime}} \delta_{\mathbf{x}, \mathbf{x}^{\prime}},
$$

where $\mathbf{x}=(\rho, \theta, \eta)$. Our notation is explained in App. A

\section{B. Mode expansion}

An expansion of the $\hat{\phi}(x)$ field in terms of transverse helicity eigenstates can be carried out using the separation of variables methods explained in Ref. 26 and further discussed App. B. The expansion is given by

$$
\hat{\phi}(x)=\sum_{\mathbf{k}, \lambda} \hat{A}_{\mathbf{k}}^{(\lambda)} \phi_{\mathbf{k}}^{(\lambda)}(x),
$$

where $\mathbf{k} \equiv\left(k_{\eta}, k_{\perp}, m, h\right)$ with

$$
\sum_{\mathbf{k}} \equiv \int_{-\infty}^{+\infty} \frac{\mathrm{d} k_{\eta}}{2 \pi} \int_{0}^{+\infty} \frac{k_{\perp} \mathrm{d} k_{\perp}}{2 \pi} \sum_{m=-\infty}^{+\infty} \sum_{h= \pm 1} .
$$

(See App. A for our notation.) Here $\lambda= \pm 1$ labels initial positive and negative energy states, $h= \pm 1$ labels the transverse helicity of the state, and $m$ the value of the $z$-component of the angular momentum operator. The time-dependent spinor mode functions $\phi_{\mathbf{k}}^{(\lambda)}(x)$ are given by

$$
\phi_{\mathbf{k}}^{(\lambda)}(x) \equiv e^{i k_{\eta} \eta}\left(\begin{array}{l}
\phi_{(+) ; k}^{(\lambda)}(\tau) \chi_{k_{m},+h}(\rho, \theta) \\
\phi_{(-) ; k}^{(\lambda)}(\tau) \chi_{k_{m},-h}(\rho, \theta)
\end{array}\right) .
$$

where $k \equiv\left(k_{\eta}, k_{\perp}, h\right)$ and $k_{m}=\left(k_{\perp}, m\right)$, and where the transverse helicity eigenvectors $\chi_{k_{m}, h}(\rho, \theta)$ are given by

$$
\chi_{k_{m}, h}(\rho, \theta)=\frac{e^{i(m+1 / 2) \theta}}{\sqrt{2}}\left(\begin{array}{c}
J_{m}\left(k_{\perp} \rho\right) \\
-h J_{m+1}\left(k_{\perp} \rho\right)
\end{array}\right) .
$$

In App. B in Eqs. B23 and B24, we show that they are orthogonal and complete.

The $\phi_{ \pm ; k}^{(\lambda)}(\tau)$ mode functions form a two-dimensional spinor,

$$
\phi_{k}^{(\lambda)}(\tau)=\left(\begin{array}{l}
\phi_{(+) ; k}^{(\lambda)}(\tau) \\
\phi_{(-) ; k}^{(\lambda)}(\tau)
\end{array}\right)
$$

which satisfies the equations of motion

$$
i \partial_{\tau} \phi_{k}^{(\lambda)}(\tau)=H_{k}(\tau) \phi_{k}^{(\lambda)}(\tau),
$$

where the Hermitian matrix $H_{k}(\tau)$ satisfies:

$$
\begin{aligned}
H_{k}(\tau) & =\left(\begin{array}{cc}
+M & \pi_{\eta}(\tau)-i h k_{\perp} \\
\pi_{\eta}(\tau)+i h k_{\perp} & -M
\end{array}\right) \\
& =\mathbf{k}_{k}(\tau) \cdot \boldsymbol{\sigma},
\end{aligned}
$$

with $\pi_{\eta}(\tau)=\left[k_{\eta}-e A(\tau)\right] / \tau$ the kinetic momentum, and where

$$
\mathbf{k}_{k}(\tau)=\pi_{\eta}(\tau) \hat{\mathbf{e}}_{1}+h k_{\perp} \hat{\mathbf{e}}_{2}+M \hat{\mathbf{e}}_{3} .
$$

We define a density matrix $\rho_{k}^{(\lambda)}(\tau)$ and "polarization" vector $\mathbf{P}_{k}^{(\lambda)}(\tau)$ by

$$
\rho_{k}^{(\lambda)}(\tau)=\phi_{k}^{(\lambda)}(\tau) \phi_{k}^{(\lambda) \dagger}(\tau)=\frac{1}{2}\left[1+\mathbf{P}_{k}^{(\lambda)}(\tau) \cdot \boldsymbol{\sigma}\right],
$$


so that from Eq. 2.35, the polarization vector satisfies:

$$
\partial_{\tau} \mathbf{P}_{k}^{(\lambda)}(\tau)=2 \mathbf{k}_{k}(\tau) \times \mathbf{P}_{k}^{(\lambda)}(\tau)
$$

We find an adiabatic expansion to second order of the polarization vector in App. C. Since $H_{k}(\tau)$ in 2.36 is Hermitian, the length of the spinors $\phi_{k}^{(\lambda)}(\tau)$ is conserved

$$
\partial_{\tau}\left[\phi_{k}^{(\lambda) \dagger}(\tau) \phi_{k}^{\left(\lambda^{\prime}\right)}(\tau)\right]=0
$$

So if we choose the two spinors labeled by $\lambda$ to be orthogonal at $\tau=\tau_{0}$, then they remain orthogonal for all $\tau$. In Sec. II C below we do this, so we can assume that these spinors are orthogonal and complete for all values of $\tau$

$$
\begin{aligned}
\phi_{k}^{(\lambda) \dagger}(\tau) \phi_{k}^{\left(\lambda^{\prime}\right)}(\tau) & =\delta_{\lambda, \lambda^{\prime}}, \\
\sum_{\lambda= \pm} \phi_{k}^{(\lambda)}(\tau) \phi_{k}^{(\lambda) \dagger}(\tau) & =1 .
\end{aligned}
$$

Probability conservation also requires that the polarization vector $\mathbf{P}_{k}^{(\lambda)}(\tau)$ for both of these solutions to remain on the unit sphere for all time $\tau$.

Using the orthogonal relations $\mathrm{B} 23$ and $2.41 \mathrm{a}$, we can invert expansion 2.30 to obtain for any time $\tau$,

$$
\hat{A}_{\mathbf{k}}^{(\lambda)}=\sum_{\mathbf{x}} \phi_{\mathbf{k}}^{(\lambda) \dagger}(x) \hat{\phi}(x)
$$

where our notation is explained in App. A. Using (2.29, the mode operators obey the anticommutation relation

$$
\left\{\hat{A}_{\mathbf{k}}^{(\lambda)}, \hat{A}_{\mathbf{k}^{\prime}}^{\left(\lambda^{\prime}\right) \dagger}\right\}=\delta_{\lambda, \lambda^{\prime}} \delta_{\mathbf{k}, \mathbf{k}^{\prime}} .
$$

It is traditional to define separate positive and negative energy mode operators by setting

$$
\hat{A}_{\mathbf{k}}^{(+)}=\hat{a}_{\mathbf{k}}, \quad \text { and } \quad \hat{A}_{\mathbf{k}}^{(-)}=\hat{b}_{-\mathbf{k}}^{\dagger} .
$$

We choose our initial state to be the vacuum with no particles or anti-particles present. Then

$$
\hat{a}_{\mathbf{k}}|0\rangle=0, \quad \text { and } \quad \hat{b}_{\mathbf{k}}|0\rangle=0 \text {. }
$$

This means that

$$
\left\langle\left[\hat{A}_{\mathbf{k}}^{(\lambda) \dagger}, \hat{A}_{\mathbf{k}^{\prime}}^{\left(\lambda^{\prime}\right)}\right]\right\rangle=-\lambda \delta_{\lambda, \lambda^{\prime}} \delta_{\mathbf{k}, \mathbf{k}^{\prime}}
$$

a result we will use in Sec. IID below.

\section{Initial conditions}

There have been several methods used to set initial conditions for the fermion field. We investigated two of these methods in Ref. 24 and came to the conclusion that both methods produce essentially the same results, so we choose the simpler "one-field" method here.
Near $\tau=\tau_{0} \equiv 1 / M$ where we take $A\left(\tau_{0}\right)=0$, the Hamiltonian 2.36 is approximately independent of $\tau$, $H_{k}(\tau) \approx H_{0 ; k}$, where

$$
H_{0 ; k}=M\left(\begin{array}{cc}
+1 & k_{\eta}-i h \bar{k}_{\perp} \\
k_{\eta}+i h \bar{k}_{\perp} & -1
\end{array}\right)=M \mathbf{k}_{0 ; k} \cdot \boldsymbol{\sigma}
$$

where

$$
\mathbf{k}_{0 ; k}=k_{\eta} \hat{\mathbf{e}}_{1}+h \bar{k}_{\perp} \hat{\mathbf{e}}_{2}+\hat{\mathbf{e}}_{3}
$$

with $\bar{k}_{\perp}=k_{\perp} / M$. We write the eigenvalue equation for the Hamiltonian $H_{0 ; k}$ as

$$
\begin{gathered}
H_{0 ; k} \phi_{0 ; k}^{(\lambda)}=\omega_{0 ; k}^{(\lambda)} \phi_{0 ; k}^{(\lambda)}, \\
\text { with } \omega_{0 ; k}^{(\lambda)}=\lambda M \omega_{0 ; k}, \quad \omega_{0 ; k}=\sqrt{k_{\eta}^{2}+\bar{k}_{\perp}^{2}+1},
\end{gathered}
$$

and where

$$
\begin{aligned}
& \phi_{0 ; k}^{(+)}=\sqrt{\frac{\omega_{0 ; k}+1}{2 \omega_{0 ; k}}}\left(\begin{array}{c}
1 \\
\zeta_{k}
\end{array}\right), \\
& \phi_{0 ; k}^{(-)}=\sqrt{\frac{\omega_{0 ; k}+1}{2 \omega_{0 ; k}}}\left(\begin{array}{c}
-\zeta_{k}^{*} \\
1
\end{array}\right),
\end{aligned}
$$

with $\zeta_{k}=\left(k_{\eta}+i h \bar{k}_{\perp}\right) /\left(\omega_{0 ; k}+1\right)$. We use these eigenvalues for initial values of the spinors $\phi_{k}^{(\lambda)}(\tau)$ at $\tau=\tau_{0}$,

$$
\phi_{k}^{(\lambda)}\left(\tau_{0}\right)=\phi_{0 ; k}^{(\lambda)},
$$

which defines what we call positive and negative energy solutions of the full Dirac equation. Since the initial spinors are orthogonal and complete, the full solutions of the Dirac are also orthogonal and complete. The density matrix $\rho_{0 ; k}^{(\lambda)}$ at $\tau_{0}$ is given by

$$
\rho_{0 ; k}^{(\lambda)}=\phi_{0 ; k}^{(\lambda)} \phi_{0 ; k}^{(\lambda) \dagger}=\frac{1}{2}\left[1+\mathbf{P}_{0 ; k}^{(\lambda)} \cdot \boldsymbol{\sigma}\right]
$$

where the initial polarization vector $\mathbf{P}_{0 ; k}^{(\lambda)}$ is given by

$$
\mathbf{P}_{0 ; k}^{(\lambda)}=\lambda \mathbf{k}_{0 ; k} / \omega_{0 ; k}
$$

\section{Maxwell's equation}

In boost-invariant coordinates, Maxwell's equation reads:

$$
\frac{1}{\sqrt{-g}} \partial_{\mu}\left[\sqrt{-g} F^{\mu \nu}(x)\right]=J^{\nu}(x),
$$

where $\sqrt{-g}=\rho \tau$. Now $A_{\mu}(x)=(0,0,0,-A(\tau))$, so the only non-vanishing elements of the field tensor are:

$$
F_{\tau, \eta}(x)=-F_{\eta, \tau}(x)=-\partial_{\tau} A(\tau) \equiv \tau E(\tau)
$$


This last equation defines what we call the electric field $E(\tau) \equiv-\partial_{\tau} A(\tau) / \tau$. Then using the metric $g^{\mu \nu}(x)=$ $\operatorname{diag}\left(1,-1,-1 / \rho,-1 / \tau^{2}\right)$, we get:

$$
F^{\tau, \eta}(\tau)=-F^{\eta, \tau}(\tau)=-E(\tau) / \tau,
$$

and Maxwell's equation becomes:

$$
\partial_{\tau} E(\tau)=-J(\tau) .
$$

Here we have defined a "reduced" current $J(\tau)$ by:

$$
\begin{aligned}
J(\tau) & =\frac{e \tau}{2}\left\langle\left[\hat{\bar{\psi}}(x), \tilde{\gamma}^{\eta}(\tau) \hat{\psi}(x)\right]\right\rangle \\
& =\frac{e}{2 \tau}\left\langle\left[\hat{\phi}^{\dagger}(x), \gamma^{0} \gamma^{3} \hat{\phi}(x)\right]\right\rangle,
\end{aligned}
$$

Using the field expansion (2.30) and the expectation value (2.46) of the mode operators, we find for the reduced current:

$$
\begin{aligned}
J(\tau)= & \frac{e}{2 \tau} \sum_{\mathbf{k}, \mathbf{k}^{\prime}} \sum_{\lambda, \lambda^{\prime}}\left[\phi_{\mathbf{k}}^{(\lambda) \dagger}(x) \gamma^{0} \gamma^{3} \phi_{\mathbf{k}^{\prime}}^{\left(\lambda^{\prime}\right)}(x)\right] \\
& \times\left\langle\left[\hat{A}_{\mathbf{k}}^{(\lambda) \dagger}, \hat{A}_{\mathbf{k}^{\prime}}^{\left(\lambda^{\prime}\right)}\right]\right\rangle \\
= & -\frac{e}{2 \tau} \sum_{\mathbf{k}, \lambda} \lambda\left[\phi_{\mathbf{k}}^{(\lambda) \dagger}(x) \gamma^{0} \gamma^{3} \phi_{\mathbf{k}}^{(\lambda)}(x)\right]
\end{aligned}
$$

Now since

$$
\gamma^{0} \gamma^{3}=\left(\begin{array}{cc}
0 & \sigma_{3} \\
\sigma_{3} & 0
\end{array}\right)
$$

and using the fact that $\sigma_{3} \chi_{k_{m}, h}(\rho, \theta)=\chi_{k_{m},-h}(\rho, \theta)$, and the relation

$$
\begin{aligned}
& \sum_{m=-\infty}^{+\infty} \chi_{k_{m}, h}^{\dagger}(\rho, \theta) \chi_{k_{m}, h}(\rho, \theta) \\
& \quad=\frac{1}{2} \sum_{m=-\infty}^{+\infty}\left[J_{m}^{2}\left(k_{\perp} \rho\right)+J_{m+1}^{2}\left(k_{\perp} \rho\right)\right]=1,
\end{aligned}
$$

we find from 2.32 that the reduced current can be written as

$$
\begin{aligned}
J(\tau) & =-\frac{e}{2 \tau} \sum_{k, \lambda} \lambda \operatorname{Tr}\left[\rho_{k}^{(\lambda)}(\tau) \sigma_{1}\right] \\
& =-\frac{e}{\tau} \sum_{k} P_{1 ; k_{\perp}, h}^{(+)}\left(\pi_{\eta}, \tau\right)=-e \sum_{p} P_{1 ; k_{\perp}, h}^{(+)}\left(\pi_{\eta}, \tau\right) .
\end{aligned}
$$

Here we have used the completeness statement 2.41b to write the current in terms of positive energy solutions only. In the last line, we changed integration variables from $k_{\eta}$ to $\pi_{\eta}$, using $\mathrm{d} \pi_{\eta}=\mathrm{d} k_{\eta} / \tau$, and defined $\mathbf{P}_{k_{\perp}, h}\left(\pi_{\eta}, \tau\right) \equiv \mathbf{P}_{k}(\tau)$. Maxwell's equation (2.57) becomes:

$$
\partial_{\tau} E(\tau)=e \sum_{p} P_{1 ; k_{\perp}, h}^{(+)}\left(\pi_{\eta}, \tau\right)
$$

Recall that $P_{1 ; k_{\perp}, h}^{(+)}\left(\pi_{\eta}, \tau\right)$ is the first component of the positive energy polarization vector. Eq. (2.39) with initial condition (2.53), and Eq. 2.63) need to be solved simultaneously for the system dynamics.

As it stands, the integral for the current in Eq. (2.63) diverges. We renormalize it using the adiabatic expansion of solutions of the Dirac equation we found in App. C Setting $\epsilon=1$ and substituting C21a into Eq. 2.63 gives

$$
\begin{aligned}
& \dot{E}(\tau) \\
& =e \sum_{p}\left[\frac{\pi_{\eta}}{\omega}-\left(k_{\perp}^{2}+M^{2}\right)\left(\frac{1}{4} \frac{\ddot{\pi}_{\eta}}{\omega^{5}}-\frac{5}{8} \frac{\pi_{\eta} \dot{\pi}_{\eta}^{2}}{\omega^{7}}\right)+\cdots\right]
\end{aligned}
$$

where here $\omega=\left[\pi_{\eta}^{2}+k_{\perp}^{2}+M^{2}\right]^{1 / 2}$. The dot refers to a derivative with respect to $\tau$. So the first term vanishes by symmetric integration over $\pi_{\eta}$. For the other terms, we note that

$$
\begin{aligned}
& \pi_{\eta}(\tau)=\left[k_{\eta}-e A(\tau)\right] / \tau \\
& \dot{\pi}_{\eta}(\tau)=-\pi_{\eta}(\tau) / \tau+e E(\tau) \\
& \ddot{\pi}_{\eta}(\tau)=2 \pi_{\eta}(\tau) / \tau^{2}-e E(\tau) / \tau+e \dot{E}(\tau) .
\end{aligned}
$$

So the only terms which survive in $(2.64)$ are

$$
\begin{aligned}
\dot{E}(\tau) & =2 e \int_{0}^{\Lambda} \frac{k_{\perp}\left(k_{\perp}^{2}+M^{2}\right) \mathrm{d} k_{\perp}}{2 \pi} \int_{-\infty}^{+\infty} \frac{\mathrm{d} \pi_{\eta}}{2 \pi} \\
& \times\left[\frac{e E(\tau)}{\tau}\left(-\frac{1}{4 \omega^{5}}+\frac{5 \pi_{\eta}^{2}}{4 \omega^{7}}\right)-\frac{e \dot{E}(\tau)}{4 \omega^{5}}+\cdots\right] .
\end{aligned}
$$

Here we have introduced a cutoff $\Lambda$ in the $k_{\perp}$ integral. Carrying out the integrals in 2.66 and moving terms proportional to $\dot{E}(\tau)$ to the left-hand-side, we find the adiabatic expansion of Maxwell's equation to be

$$
\left[1+e^{2}\left(\delta e^{2}\right)\right] \dot{E}(\tau)=e R[e A(\tau)]
$$

where $\delta e^{2}$ is given by

$$
\delta e^{2}=\frac{1}{6 \pi^{2}} \ln [\Lambda / M],
$$

and $R[e A(\tau)]$ is a finite functional of the product $e A(\tau)$, or derivatives of this quantity. We define the renormalized charge $e_{r}$ by

$$
e_{r}^{2}=\frac{e^{2}}{1+e^{2}\left(\delta e^{2}\right)} .
$$

Then since $e A(\tau)=e_{r} A_{r}(\tau)$, the adiabatic expansion of Maxwell's equation 2.67 reduces to

$$
\dot{E}_{r}(\tau)=e_{r} R\left[e_{r} A_{r}(\tau)\right],
$$

which is now finite. We conclude that we can regularize Maxwell's equation by subtracting from the integrand 
the adiabatic expansion of $P_{1 ; k_{\perp}, h}^{(+)}\left(\pi_{\eta}, \tau\right)$ and in addition renormalizing the charge. This gives the equation

$$
\begin{gathered}
\partial_{\tau} E(\tau)=\frac{e}{1+e^{2}\left(\delta e^{2}\right)} \sum_{p}^{\Lambda}\left[P_{1 ; k_{\perp}, h}^{(+)}\left(\pi_{\eta}, \tau\right)\right. \\
\left.-\frac{\pi_{\eta}}{\omega}+\frac{e E(\tau)}{\tau}\left(k_{\perp}^{2}+M^{2}\right)\left(\frac{1}{4 \omega^{5}}-\frac{5 \pi_{\eta}^{2}}{4 \omega^{7}}\right)\right] .
\end{gathered}
$$

\section{ENERGY-MOMENTUM TENSOR}

In the boost-invariant coordinate system, the average value of the total energy-momentum tensor is given by Eqs. (4.1) and (4.2) of Ref. 23, and is the sum of two terms (notice sign convention)

$$
\begin{aligned}
T_{\mu \nu} & =T_{\mu \nu}^{\text {matter }}+T_{\mu \nu}^{\text {field }} \\
& =\operatorname{diag}\left(\mathcal{E}, \mathcal{P}_{\perp}, \rho^{2} \mathcal{P}_{\theta}, \tau^{2} \mathcal{P}_{\eta}\right)
\end{aligned}
$$

where the matter and field contributions are given by

$$
\begin{aligned}
T_{\mu \nu}^{\text {mat }} & =\frac{1}{4}\left\langle\left[\hat{\bar{\psi}}(x), \tilde{\gamma}_{(\mu}(x)\left(i D_{\nu)} \hat{\psi}(x)\right)\right]+\text { h.c. }\right\rangle \\
T_{\mu \nu}^{\text {field }} & =g_{\mu \nu} \frac{1}{4} F^{\alpha \beta} F_{\alpha \beta}+F_{\mu \alpha} g^{\alpha \beta} F_{\beta \nu} .
\end{aligned}
$$

Here $D_{\mu}=\partial_{\mu}+i e A_{\mu}(x)$ and the subscript notation $(\mu, \nu)$ means to symmetrize the term. From our results for the field tensor in Eq. 2.55 in Sec. II D the field part of the energy-momentum tensor is given by

$$
T_{\mu \nu}^{\text {field }}=\frac{1}{2} \operatorname{diag}\left(E^{2}, E^{2}, \rho^{2} E^{2},-\tau^{2} E^{2}\right) .
$$

We denote the matter part of the energy-momentum tensor as:

$$
T_{\mu \nu}^{\text {matter }}=\operatorname{diag}\left(\varepsilon, p_{\perp}, \rho^{2} p_{\theta}, \tau^{2} p_{\eta}\right)
$$

Because of the conventions adapted in Eq. 3.1, the total energy and pressures are obtained by adding a factor of $\pm E^{2} / 2$ to the matter terms.

For the matter field, we first note that $D_{\mu} \hat{\psi}(x)=$ $S(x) \nabla_{\mu} \hat{\phi}(x) / \sqrt{\tau}$, where $\nabla_{\mu}=\partial_{\mu}+\Pi_{\mu}(x)+i e A_{\mu}(x)$ is the covariant derivative defined in Eq. 2.23. So using the use the notation in App. A, the field expansion 2.30 , and the expectation value 2.46) of the mode operators, the diagonal components of the matter energymomentum tensor (3.2a) are given by (no sum over $\mu$ )

$$
\begin{aligned}
& T_{\mu \mu}^{\text {mat }}=\frac{1}{4}\left\langle\left[\left(\frac{\hat{\phi}^{\dagger}(x)}{\sqrt{\tau}}\right) \gamma^{0}, \bar{\gamma}_{\mu}(x)\left\{i \nabla_{\mu}\left(\frac{\hat{\phi}(x)}{\sqrt{\tau}}\right)\right\}\right]+\left[\left\{i \bar{\nabla}_{\mu}\left(\frac{\hat{\phi}(x)}{\sqrt{\tau}}\right)\right\}^{\dagger} \gamma^{0}, \bar{\gamma}_{\mu}(x)\left(\frac{\hat{\phi}(x)}{\sqrt{\tau})]\rangle}(3.5)\right.\right.\right. \\
& =\frac{1}{4} \sum_{\mathbf{k}, \mathbf{k}^{\prime}} \sum_{\lambda, \lambda^{\prime}}\left[\left(\frac{\hat{\phi}_{\mathbf{k}}^{(\lambda) \dagger}(x)}{\sqrt{\tau}}\right) \gamma^{0} \bar{\gamma}_{\mu}(x)\left\{i \nabla_{\mu}\left(\frac{\hat{\phi}_{\mathbf{k}^{\prime}}^{\left(\lambda^{\prime}\right)}(x)}{\sqrt{\tau}}\right)\right\}+\left\{i \bar{\nabla}_{\mu}\left(\frac{\hat{\phi}_{\mathbf{k}}^{(\lambda)}(x)}{\sqrt{\tau}}\right)\right\}^{\dagger} \gamma^{0} \bar{\gamma}_{\mu}(x)\left(\frac{\hat{\phi}_{\mathbf{k}^{\prime}}^{\left(\lambda^{\prime}\right)}(x)}{\sqrt{\tau}}\right)\right]\left\langle\left[\hat{A}_{\mathbf{k}}^{(\lambda) \dagger}, \hat{A}_{\mathbf{k}^{\prime}}^{\left(\lambda^{\prime}\right)}\right]\right\rangle \\
& =\frac{-1}{4} \sum_{\mathbf{k}, \lambda} \lambda\left[\left(\frac{\hat{\phi}_{\mathbf{k}}^{(\lambda) \dagger}(x)}{\sqrt{\tau}}\right)\left\{i \gamma^{0} \bar{\gamma}_{\mu}(x) \nabla_{\mu}\left(\frac{\hat{\phi}_{\mathbf{k}}^{(\lambda)}(x)}{\sqrt{\tau}}\right)\right\}+\left\{i \gamma^{0} \bar{\gamma}_{\mu}(x) \bar{\nabla}_{\mu}\left(\frac{\hat{\phi}_{\mathbf{k}}^{(\lambda)}(x)}{\sqrt{\tau}}\right)\right\}^{\dagger}\left(\frac{\hat{\phi}_{\mathbf{k}}^{(\lambda)}(x)}{\sqrt{\tau}}\right)\right],
\end{aligned}
$$

where we used the fact that $\bar{\gamma}_{\mu}(x)$ anticommutes with $\Pi_{\mu}(x)$, and the relation $\gamma^{0} \Pi_{\mu}^{\dagger}(x) \gamma^{0}=-\Pi_{\mu}(x)$. Here we have defined the covariant derivatives

$$
\begin{gathered}
\nabla_{\mu}=\partial_{\mu}+\Pi_{\mu}(x)+i e A_{\mu}(x), \\
\bar{\nabla}_{\mu}=\partial_{\mu}-\Pi_{\mu}(x)+i e A_{\mu}(x) .
\end{gathered}
$$

\section{A. Energy Density}

For matter energy density term, $\nabla_{0}=\partial_{\tau}$ and $\bar{\gamma}_{\tau}=$ $\bar{\gamma}^{\tau}=\gamma^{0}$, so using (3.5), we find

$$
\begin{aligned}
\varepsilon(\tau) & =-\frac{i}{4 \tau} \sum_{\mathbf{k}, \lambda} \lambda\left[\phi_{\mathbf{k}}^{(\lambda) \dagger}(x) \overleftrightarrow{\partial_{\tau}} \phi_{\mathbf{k}}^{(\lambda)}(x)\right] \\
& =-\frac{1}{2 \tau} \sum_{k, \lambda} \operatorname{Tr}\left[\rho_{\mathbf{k}}^{(\lambda)}(\tau) H_{\mathbf{k}}(\tau)\right] \\
& =-\sum_{p} \mathbf{k}_{k_{\perp}, h}\left(\pi_{\eta}\right) \cdot \mathbf{P}_{k_{\perp}, h}^{(+)}\left(\pi_{\eta}, \tau\right) .
\end{aligned}
$$

So from 3.3, the total energy density is given by

$$
\mathcal{E}=-\sum_{p} \mathbf{k}_{k_{\perp}, h}\left(\pi_{\eta}\right) \cdot \mathbf{P}_{k_{\perp}, h}^{(+)}\left(\pi_{\eta}, \tau\right)+\frac{E^{2}}{2} .
$$


As it stands, the integral for the energy density in Eq. 3.8 diverges. We find the form of theses divergences by substituting the adiabatic expansion given in Eq. C20 and introducing a cutoff $\Lambda_{\eta}$ in the $\pi_{\eta}$ integral. This gives a adiabatic approximation to the energy density of

$$
\mathcal{E}_{a}=\frac{E^{2}}{2}-\sum_{p}^{\Lambda}\left[\omega-\frac{\left(k_{\perp}^{2}+M^{2}\right)}{8} \frac{\dot{\pi}_{\eta}^{2}}{\omega^{5}} \cdots\right] .
$$

Using Eqs. 2.65, due to the symmetric integration over $\pi_{\eta}$, the only terms that survive are

$$
\begin{aligned}
& \mathcal{E}_{a}=\frac{E^{2}}{2}-\sum_{p}^{\Lambda}\left[\omega-\frac{k_{\perp}^{2}+M^{2}}{8}\left(\frac{1}{\tau^{2}} \frac{\pi_{\eta}^{2}}{\omega^{5}}+\frac{e^{2} E^{2}}{\omega^{5}}\right)+\cdots\right] \\
& =\left[1+e^{2}\left(\delta e^{2}\right)\right] \frac{E^{2}}{2}+\frac{1}{12}\left(\frac{\Lambda}{2 \pi \tau}\right)^{2}-\sum_{p}^{\Lambda}(\omega+\cdots) .
\end{aligned}
$$

The first term renormalizes the field

$$
\left[1+e^{2}\left(\delta e^{2}\right)\right] \frac{E^{2}}{2}=\frac{e^{2} E^{2}}{2 e_{r}^{2}}=\frac{E_{r}^{2}}{2} .
$$

The second term contributes to the cosmological constant, as we will see later. The third term is related to the zero-point energy of pairs of fermions. We regularize the energy density by computing the difference between $\mathcal{E}$ and $\mathcal{E}_{a}, \mathcal{E}^{\text {sub }}=\mathcal{E}-\mathcal{E}_{a}$, which is now finite.

\section{B. Transverse pressure}

For the matter transverse pressure term, we have $\bar{\gamma}_{\rho}=$ $-\bar{\gamma}^{\rho}=-\gamma^{1}$, and $\nabla_{\rho}=\partial_{\rho}$, so from (3.5), we find:

$$
p_{\perp}(\tau)=-\frac{i}{4 \tau} \sum_{\mathbf{k}, \lambda} \lambda\left[\phi_{\mathbf{k}}^{(\lambda) \dagger}(x) \gamma^{0} \gamma^{1} \overleftrightarrow{\partial_{\rho}} \phi_{\mathbf{k}}^{(\lambda)}(x)\right]
$$

So here we will need to find

$$
\begin{aligned}
& \sum_{m=-\infty}^{+\infty} \chi_{k_{\perp}, m, h}^{\dagger}(\rho) \sigma_{x} \overleftrightarrow{\partial_{\rho}} \chi_{k_{\perp}, m,-h}(\rho) \\
& \quad=h \sum_{m=-\infty}^{+\infty} J_{m}\left(k_{\perp} \rho\right) \overleftrightarrow{\partial_{\rho}} J_{m+1}\left(k_{\perp} \rho\right)=h k_{\perp}
\end{aligned}
$$

where we have used the relation

$$
2 J_{m}^{\prime}(z)=J_{m-1}(z)-J_{m+1}(z) .
$$

Then 3.11 becomes

$$
\begin{aligned}
p_{\perp}(\tau) & =\frac{1}{\tau} \sum_{k} \frac{h k_{\perp}}{2}\left[\phi_{k}^{(+) \dagger}(\tau) \sigma_{y} \phi_{k}^{(+)}(\tau)\right] \\
& =\sum_{p} \frac{h k_{\perp}}{2} P_{2 ; k_{\perp}, h}^{(+)}\left(\pi_{\eta}, \tau\right)
\end{aligned}
$$

and from 3.3 , the total transverse pressure is given by

$$
\mathcal{P}_{\perp}=\sum_{p} \frac{h k_{\perp}}{2} P_{2 ; k_{\perp}, h}^{(+)}\left(\pi_{\eta}, \tau\right)+\frac{E^{2}}{2} .
$$

In order to study the divergences in the transverse pressure, we substitute the adiabatic expansion $\mathrm{C} 21 \mathrm{~b}$ into 3.13. This gives

$$
\begin{aligned}
\mathcal{P}_{a ; \perp}=\frac{E^{2}}{2} & +\sum_{p}^{\Lambda}\left[\frac{k_{\perp}^{2}}{2 \omega}-\frac{h k_{\perp} M \dot{\pi}_{\eta}}{4 \omega^{3}}\right. \\
& \left.+\frac{k_{\perp}^{2}}{2}\left(-\frac{1}{8} \frac{\dot{\pi}_{\eta}^{2}}{\omega^{5}}+\frac{1}{4} \frac{\pi_{\eta} \ddot{\pi}_{\eta}}{\omega^{5}}-\frac{5}{8} \frac{\pi_{\eta}^{2} \dot{\pi}_{\eta}^{2}}{\omega^{7}}\right)+\cdots\right] .
\end{aligned}
$$

The second term in the above sum over $p$ is odd in $h$ and therefore vanishes. From Eqs. 2.65, the only terms that survive the $\pi_{\eta}$ integration are

$$
\begin{aligned}
\mathcal{P}_{a ; \perp}= & \frac{E^{2}}{2}+\sum_{p}^{\Lambda}\left\{\frac{k_{\perp}^{2}}{2 \omega}-\frac{k_{\perp}^{2}}{2}\left[-\frac{1}{\tau^{2}}\left(\frac{3}{8} \frac{\pi_{\eta}^{2}}{\omega^{5}}-\frac{5}{8} \frac{\pi_{\eta}^{4}}{\omega^{7}}\right)\right.\right. \\
& \left.\left.-e^{2} E^{2}\left(\frac{1}{8} \frac{1}{\omega^{5}}+\frac{5}{8} \frac{\pi_{\eta}^{2}}{\omega^{7}}\right)\right]+\cdots\right\} \\
= & {\left[1+e^{2}\left(\delta e^{2}\right)\right] \frac{E^{2}}{2}+\sum_{p}^{\Lambda}\left(\frac{k_{\perp}^{2}}{2 \omega}+\cdots\right) }
\end{aligned}
$$

From 3.10 , the first term renormalizes the electric field. The term proportional to $1 / \tau^{2}$ vanishes. Again, the transverse pressure is regularized by subtracting the adiabatic expression from the divergent one, $\mathcal{P}_{\perp}^{\text {sub }}=\mathcal{P}_{\perp}-$ $\mathcal{P}_{a ; \perp}$.

\section{Shear Pressure}

For the shear pressure term, we have $\bar{\gamma}_{\theta}=-\rho^{2} \bar{\gamma}^{\theta}=$ $-\rho \gamma^{2}$ and $\Pi_{\theta}=\gamma^{1} \gamma^{2}$. The covariant derivatives (3.6) are given by

$$
\begin{aligned}
& \gamma^{0} \gamma^{2} \nabla_{\theta}=\gamma^{0} \gamma^{2} \partial_{\theta}+\gamma^{0} \gamma^{1} / 2 \\
& \gamma^{0} \gamma^{2} \bar{\nabla}_{\eta}=\gamma^{0} \gamma^{2} \partial_{\theta}-\gamma^{0} \gamma^{1} / 2
\end{aligned}
$$

where

$$
\gamma^{0} \gamma^{1}=\left(\begin{array}{cc}
0 & \sigma_{x} \\
\sigma_{x} & 0
\end{array}\right), \quad \gamma^{0} \gamma^{2}=\left(\begin{array}{cc}
0 & \sigma_{y} \\
\sigma_{y} & 0
\end{array}\right) .
$$

So from 3.5, we find

$$
\begin{gathered}
p_{\theta}(\tau)=\frac{-i}{4 \rho \tau} \sum_{\mathbf{k}, \lambda} \lambda\left\{\phi_{\mathbf{k}}^{(\lambda) \dagger}(x)\left[\left(\gamma^{0} \gamma^{2} \partial_{\theta}+\gamma^{0} \gamma^{1} / 2\right) \phi_{\mathbf{k}}^{(\lambda)}(x)\right]\right. \\
\left.+\left[\left(\gamma^{0} \gamma^{2} \partial_{\theta}-\gamma^{0} \gamma^{1} / 2\right) \phi_{\mathbf{k}}^{(\lambda)}(x)\right]^{\dagger} \phi_{\mathbf{k}}^{(\lambda)}(x)\right\} .
\end{gathered}
$$


So here we need to compute

$$
\begin{aligned}
& \sum_{m=-\infty}^{+\infty} \chi_{k_{m}, h}^{\dagger}(\theta, \rho)\left(\sigma_{y} \partial_{\theta} \pm \sigma_{x} / 2\right) \chi_{k_{m},-h}(\theta, \rho) \\
& =\frac{h}{2} \sum_{m=-\infty}^{+\infty}(2 m+1) J_{m}\left(k_{\perp} \rho\right) J_{m+1}\left(k_{\perp} \rho\right)=\frac{h k_{\perp} \rho}{2}
\end{aligned}
$$

where we have used the relation

$$
2 m J_{m}(z)=z\left[J_{m+1}(z)+J_{m-1}(z)\right] .
$$

Then 3.16 becomes

$$
\begin{aligned}
p_{\theta}(\tau) & =\frac{1}{\tau} \sum_{k} \frac{h k_{\perp}}{2}\left[\phi_{k}^{(+) \dagger}(\tau) \sigma_{y} \phi_{k}^{(+)}(\tau)\right] \\
& =\sum_{p}\left(\frac{h k_{\perp}}{2}\right) P_{2 ; k_{\perp}, h}^{(+)}\left(\pi_{\eta}, \tau\right)
\end{aligned}
$$

adding this to the shear pressure of the field, we find

$$
\mathcal{P}_{\theta}=\sum_{p} \frac{h k_{\perp}}{2} P_{2 ; k_{\perp}, h}^{(+)}\left(\pi_{\eta}, \tau\right)+\frac{E^{2}}{2} .
$$

Note that $\mathcal{P}_{\theta}=\mathcal{P}_{\perp}$. The shear pressure is renormalized exactly like the transverse pressure.

\section{Longitudinal pressure}

For the longitudinal pressure $(\mu=\eta), A_{\eta}(x)=-A(\tau)$ and $\bar{\gamma}_{\eta}=-\tau^{2} \bar{\gamma}^{\eta}=-\tau \gamma^{3}$. The covariant derivatives (3.6) are given by

$$
\begin{aligned}
& i \gamma^{0} \gamma^{3} \nabla_{\eta}=\gamma^{0} \gamma^{3}\left[i \partial_{\eta}+e A(\tau)\right]+\gamma^{5} / 2, \\
& i \gamma^{0} \gamma^{3} \bar{\nabla}_{\eta}=\gamma^{0} \gamma^{3}\left[i \partial_{\eta}+e A(\tau)\right]-\gamma^{5} / 2,
\end{aligned}
$$

where

$$
\gamma^{5}=i \gamma^{0} \gamma^{1} \gamma^{2} \gamma^{3}=\left(\begin{array}{cc}
0 & 1 \\
1 & 0
\end{array}\right), \quad \gamma^{0} \gamma^{3}=\left(\begin{array}{cc}
0 & \sigma_{z} \\
\sigma_{z} & 0
\end{array}\right)
$$

Here $\gamma^{5}$ flips the upper and lower components of the spinor, which leads to the equation

$$
\begin{aligned}
& \sum_{m=-\infty}^{+\infty} \chi_{k_{m}, h}^{\dagger}(\rho, \theta) \chi_{k_{m},-h}(\rho, \theta) \\
& \quad=\frac{1}{2} \sum_{m=-\infty}^{+\infty}\left[J_{m}^{2}\left(k_{\perp} \rho\right)-J_{m+1}^{2}\left(k_{\perp} \rho\right)\right]=0 .
\end{aligned}
$$

So terms proportional to $\gamma^{5}$ vanish. Then from 3.5 , we are left with

$$
\begin{aligned}
p_{\eta}(\tau)= & \frac{1}{4 \tau^{2}} \sum_{\mathbf{k}, \lambda} \lambda\left\{\phi_{\mathbf{k}}^{(\lambda) \dagger}(x)\left[\gamma^{0} \gamma^{3}\left(i \partial_{\eta}+e A(\tau)\right) \phi_{\mathbf{k}}^{(\lambda)}(x)\right]\right. \\
& \left.\quad+\left[\gamma^{0} \gamma^{3}\left(i \partial_{\eta}+e A(\tau)\right) \phi_{\mathbf{k}}^{(\lambda)}(x)\right]^{\dagger} \phi_{\mathbf{k}}^{(\lambda)}(x)\right\} \\
= & \frac{-1}{\tau^{2}} \sum_{k}\left[k_{\eta}-e A(\tau)\right] P_{1 ; k}(\tau) \\
= & -\sum_{p} \pi_{\eta} P_{1 ; k_{\eta}, h}\left(\pi_{\eta}, \tau\right) .
\end{aligned}
$$

Adding the field pressure, we find for the total longitudinal pressure

$$
\mathcal{P}_{\eta}=-\sum_{p} \pi_{\eta} P_{1 ; k_{\eta}, h}\left(\pi_{\eta}, \tau\right)-\frac{E^{2}}{2} .
$$

From Eq. C21a, the adiabatic expansion of the longitudinal pressure is given by

$$
\begin{aligned}
& \mathcal{P}_{a ; \eta}=-\frac{E^{2}}{2} \\
& -\sum_{p}^{\Lambda}\left[\frac{\pi_{\eta}^{2}}{\omega}-\left(k_{\perp}^{2}+M^{2}\right)\left(\frac{1}{4} \frac{\pi_{\eta} \ddot{\pi}_{\eta}}{\omega^{5}}-\frac{5}{8} \frac{\pi_{\eta}^{2} \dot{\pi}_{\eta}^{2}}{\omega^{7}}\right)+\cdots\right] .
\end{aligned}
$$

From Eqs. 2.65, the only terms that survive the $\pi_{\eta}$ integration are

$$
\begin{gathered}
\mathcal{P}_{a ; \eta}=-\frac{E^{2}}{2}-\sum_{p}^{\Lambda}\left[\frac{\pi_{\eta}^{2}}{\omega}+\frac{5}{8} e^{2} E^{2}\left(k_{\perp}^{2}+M^{2}\right) \frac{\pi_{\eta}^{2}}{\omega^{7}}\right. \\
\left.-\frac{\left(k_{\perp}^{2}+M^{2}\right)}{2 \tau^{2}}\left(\frac{\pi_{\eta}^{2}}{\omega^{5}}-\frac{5}{4} \frac{\pi_{\eta}^{4}}{\omega^{7}}\right)+\cdots\right] \quad(3.23) \\
=-\left[1+e^{2}\left(\delta e^{2}\right)\right] \frac{E^{2}}{2}+\frac{1}{12}\left(\frac{\Lambda}{2 \pi \tau}\right)^{2}-\sum_{p}^{\Lambda}\left(\frac{\pi_{\eta}^{2}}{\omega}+\cdots\right) .
\end{gathered}
$$

Again, the first term renormalizes the electric field and the second term renormalizes the cosmological constant. The finite part of the longitudinal pressure is given by $\mathcal{P}_{\eta}^{\text {sub }}=\mathcal{P}_{\eta}-\mathcal{P}_{a ; \eta}$, as before.

\section{E. Conservation equations}

The covariant derivative of the energy-momentum tensor in boost-invariant coordinates is conserved

$$
T_{; \mu}^{\mu \nu}=\partial_{\mu} T^{\mu \nu}+\Gamma_{\mu \sigma}^{\mu} T^{\sigma \nu}+\Gamma_{\mu \sigma}^{\nu} T^{\mu \sigma}=0 .
$$

The only nonzero Christoffel symbols are given in Eq. 2.25). There are only two conservation equations that result from Eq. 3.24). For $\nu=\tau$, 3.24) reduces to

$$
\begin{aligned}
& \partial_{\tau} T^{\tau \tau}+T^{\tau \tau} / \tau+\tau T^{\eta \eta}=0, \\
& \text { or } \quad \partial_{\tau}(\tau \mathcal{E})+\mathcal{P}_{\eta}=0 .
\end{aligned}
$$


Using the equation of motion 2.39 and Maxwell's equation (2.63), one can show that Eq. (3.25) is automatically satisfied.

The conservation equation for $\nu=\rho$ amounts to a relation between the transverse and shear pressures. We find that $\mathcal{P}_{\perp}=\mathcal{P}_{\theta}$, which is satisfied by our expression in Eqs. (3.13) and (3.18). For the one-dimensional boost invariant expansion we had instead for the equation for the energy density,

$$
\partial_{\tau}(\tau \mathcal{E})+\mathcal{P}=0 .
$$

\section{QUASIPARTICLE PHASE SPACE DISTRIBUTION FUNCTIONS}

For the problem at hand, particle production from classical electric fields, it is possible to introduce an interpolating number density via a Bogoliubov transformation that is an adiabatic invariant. This was done previously [21, 22, 23, 24]). However, when we consider the fully interacting case with quantum gauge fields then one needs to resort to an "effective" quasiparticle distribution function that allows one to reproduce the expectation value of the current and the energy-momentum tensor. That is, we want to determine an effective distribution function $f(x, k)$ in analogy with relativistic kinetic theory (see for example Refs. 27, 28]) such that

$$
\left\langle J^{\mu}\right\rangle=e \int \mathrm{D} k k^{\mu} f(x, k)
$$

and

$$
\left\langle T^{\mu \nu}\right\rangle=\int \mathrm{D} k k^{\mu} k^{\nu} f(x, k),
$$

where

$$
\mathrm{D} k=2 r \theta\left(k^{0}\right) \delta\left(k^{2}-M^{2}\right) \frac{d^{4} k}{(2 \pi)^{3} \sqrt{-g}} .
$$

Here, $r$ is a degeneracy factor which counts the number of species. For our case of quark and anti-quark pairs with spin one-half, we have $r=4$. Hence, the renormalized comoving energy density, $\overline{\mathcal{E}}=T_{00}$, is given by

$$
\overline{\mathcal{E}}=\frac{4}{\tau} \int_{0}^{+\infty} \frac{k_{\perp} \mathrm{d} k_{\perp}}{2 \pi} \int_{-\infty}^{+\infty} \frac{\mathrm{d} k_{\eta}}{2 \pi} \omega_{k_{\perp}, k_{\eta}}(\tau) f\left(\tau, k_{\perp}, k_{\eta}\right)
$$

and will be identified with the renormalized field theory result:

$$
\mathcal{E}=-\left[\int \mathbf{k}_{k_{\perp}, h}\left(\pi_{\eta}\right) \cdot \mathbf{P}_{k_{\perp}, h}^{(+)}\left(\pi_{\eta}, \tau\right)+\frac{E^{2}}{2}\right]_{\text {ren }},
$$

where we have subtracted the divergences coming from the cosmological term and the charge renormalization. Note that when the single-particle distribution becomes independent of proper time, $\tau$, one can easily derive the

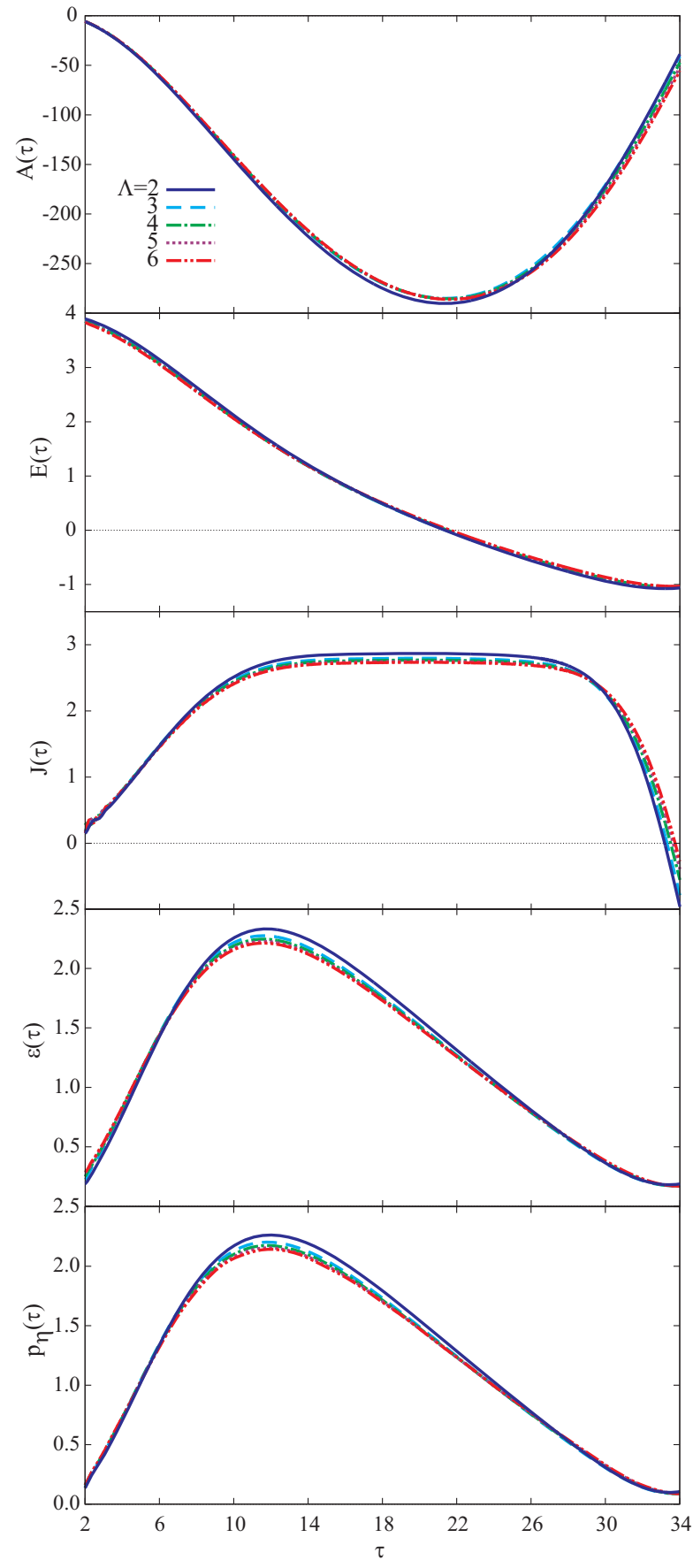

FIG. 1: (Color online) Convergence of the proper-time evolution of the electromagnetic fields, current, energy and longitudinal pressure with respect to the cutoff $\Lambda$, in $(3+1)$ dimensional QED. Here we choose $m=1, A\left(\tau_{0}\right)=0$ and $E\left(\tau_{0}\right)=4$.

conservation of energy equation in terms of the energy density and longitudinal pressure: Consider the identity

$$
\frac{4}{\tau} \int_{0}^{+\infty} \frac{k_{\perp} \mathrm{d} k_{\perp}}{2 \pi} \int_{-\infty}^{+\infty} \frac{\mathrm{d} k_{\eta}}{2 \pi} \omega_{k_{\perp}, k_{\eta}}(\tau) \frac{\partial f\left(\tau, k_{\perp}, k_{\eta}\right)}{\partial \tau}=0,
$$




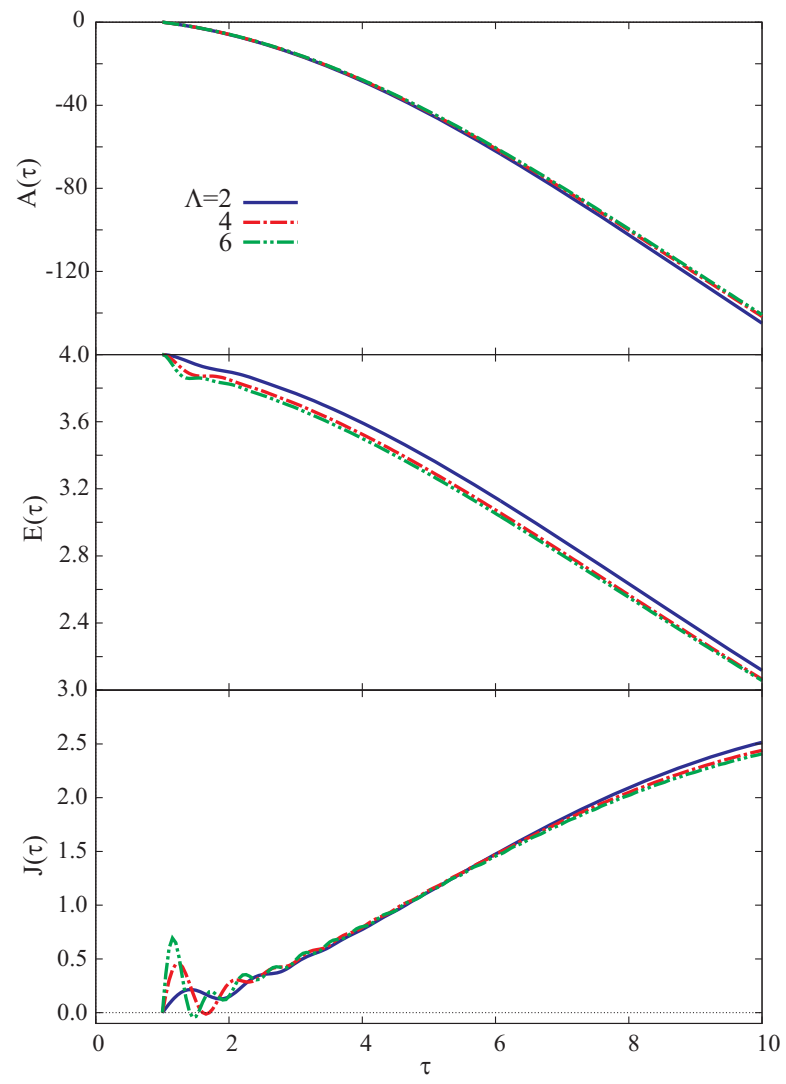

FIG. 2: (Color online) Early proper-time evolution of the electromagnetic fields and current with respect to the cutoff $\Lambda$, in $(3+1)$-dimensional QED. We note that for $\tau<5$ the results are dependent on the cutoff, whereas at later proper times the results become insensitive to the cutoff. This is an artifact of our choice of initial conditions, which are not consistent at early times with the adiabatic-expansion-based substraction scheme.

then integrate by parts to obtain

$$
\frac{\partial \overline{\mathcal{E}}}{\partial \tau}+\frac{\overline{\mathcal{E}}+\overline{\mathcal{P}}_{\eta}}{\tau}=0
$$

where the longitudinal pressure is introduced as

$$
\overline{\mathcal{P}}_{\eta}=\frac{4}{\tau} \int_{0}^{+\infty} \frac{k_{\perp} \mathrm{d} k_{\perp}}{2 \pi} \int_{-\infty}^{+\infty} \frac{\mathrm{d} k_{\eta}}{2 \pi} \frac{\left(k_{\eta} / \tau\right)^{2}}{\omega_{k_{\perp}, k_{\eta}}(\tau)} f\left(\tau, k_{\perp}, k_{\eta}\right) .
$$

The quasiparticle phase-space distribution of pairs of particles and antiparticles with a specific spin in lightcone variables is introduced as

$$
\frac{\mathrm{d}^{6} N}{\mathrm{~d}^{2} x_{\perp} \mathrm{d} \eta \mathrm{d}^{2} k_{\perp} \mathrm{d} k_{\eta}}=\frac{f\left(\tau, k_{\perp}, k_{\eta}\right)}{(2 \pi)^{3}},
$$

such that the pair density is obtained as

$$
\frac{\mathrm{d}^{3} N}{\mathrm{~d}^{2} x_{\perp} \mathrm{d} \eta}=\int_{0}^{+\infty} \frac{k_{\perp} \mathrm{d} k_{\perp}}{2 \pi} \int_{-\infty}^{+\infty} \frac{\mathrm{d} k_{\eta}}{2 \pi} f\left(\tau, k_{\perp}, k_{\eta}\right)
$$

Here, we have $\mathrm{d}^{2} x_{\perp}=\rho \mathrm{d} \rho \mathrm{d} \theta$ and $\mathrm{d}^{2} k_{\perp}=k_{\perp} \mathrm{d} k_{\perp} \mathrm{d} \phi$. For completeness, we note that in $(1+1)$ dimensions the

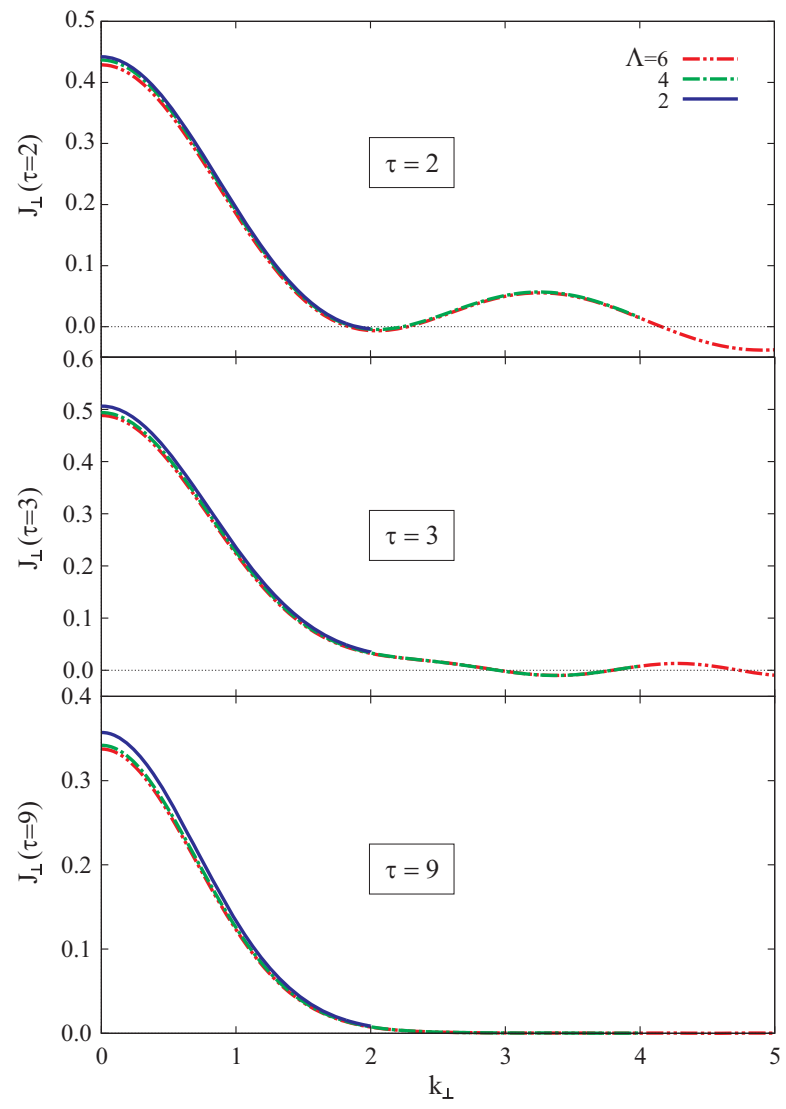

FIG. 3: (Color online) Proper-time evolution of the transverse ( $k_{\perp}$-projected) distribution of the current for $\tau$ values of 2,3 and 9 . The oscillations of the transverse distributions of the current present at early proper times are consequences of the inconsistency between the adiabatic-expansion regularization scheme and our choice of initial conditions which are designed to keep the storage and time requirements of the simulation to a minimum. At later proper times, these oscillations dampen out and eventually disappear. Therefore, the early propertime evolution should be regarded as "unphysical" and will be disregarded.

pair density reads

$$
\frac{\mathrm{d} N}{\mathrm{~d} \eta}=\int_{-\infty}^{+\infty} \frac{\mathrm{d} k_{\eta}}{2 \pi} f\left(\tau, k_{\eta}\right)
$$

When the pair distribution becomes independent of the proper time $\tau$ then we are in the "out-regime" and can stop our calculation as far as determining the particle spectra. We need to relate this quantity to the center-ofmass distribution of electrons and positrons produced by the strong electric field. We introduce the free-particle rapidity, $y=\frac{1}{2} \ln \left[\left(E+k_{z}\right) /\left(E-k_{z}\right)\right]$, and "transverse" mass, $M_{\perp}=\sqrt{k_{\perp}^{2}+m^{2}}$, by relating them to the cartesian coordinate four-momentum in the center-of-mass system, $\hat{k}^{a}=(E, \mathbf{k})$, by the relation

$$
\hat{k}^{a}=\left(M_{\perp} \cosh y, \mathbf{k}_{\perp}, M_{\perp} \sinh y\right)
$$




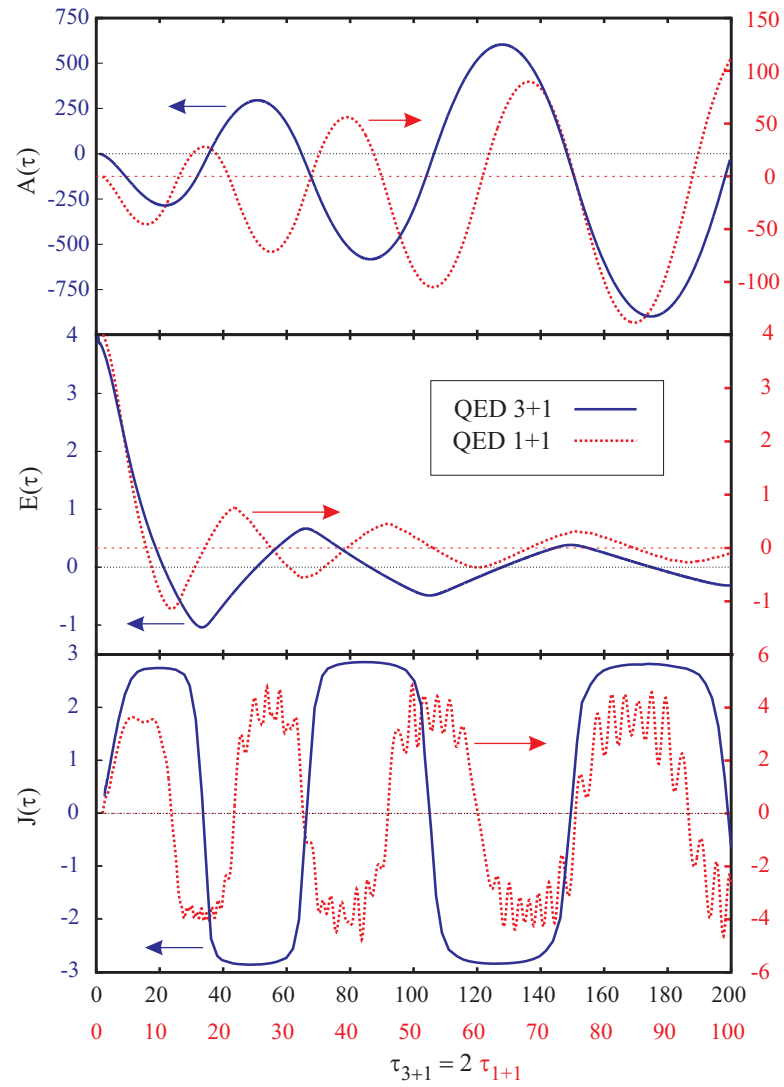

FIG. 4: (Color online) Proper-time evolution of the electromagnetic fields and current for the case of $(1+1)$ - and (3+1)-dimensional QED, respectively. Here we choose $m=1$, $A\left(\tau_{0}\right)=0$ and $E\left(\tau_{0}\right)=4$.

The boost that takes one from the center-of-mass coordinates to the comoving frame, where the energy momentum tensor is diagonal, is given by $\tanh \eta=v=z / t$, so that one can define the "fluid" four-velocity in the center-of-mass frame as

$$
u^{a}=(\cosh \eta, 0,0, \sinh \eta) .
$$

It is important to relate the momenta canonical to $\eta$ and $\tau$ to the center-of-mass variables. In the out regime we can identify these canonical momenta from the free particle (1+1)-dimensional Lagrangian in covariant form. We show now that

$$
\tau=\sqrt{t^{2}-z^{2}}, \quad \eta=\frac{1}{2} \ln \left(\frac{t+z}{t-z}\right),
$$

have as their canonical momenta

$$
k_{\tau}=E t / \tau-k_{z} z / \tau, \quad k_{\eta}=-E z+t k_{z} .
$$

Consider the metric $d s^{2}=d \tau^{2}-\tau^{2} d \eta^{2}$ and the free particle Lagrangian in $(1+1)$ dimensions

$$
L=\frac{M}{2} g_{\mu \nu} \frac{\mathrm{d} x^{\mu}}{\mathrm{d} s} \frac{\mathrm{d} x^{\nu}}{\mathrm{d} s} .
$$

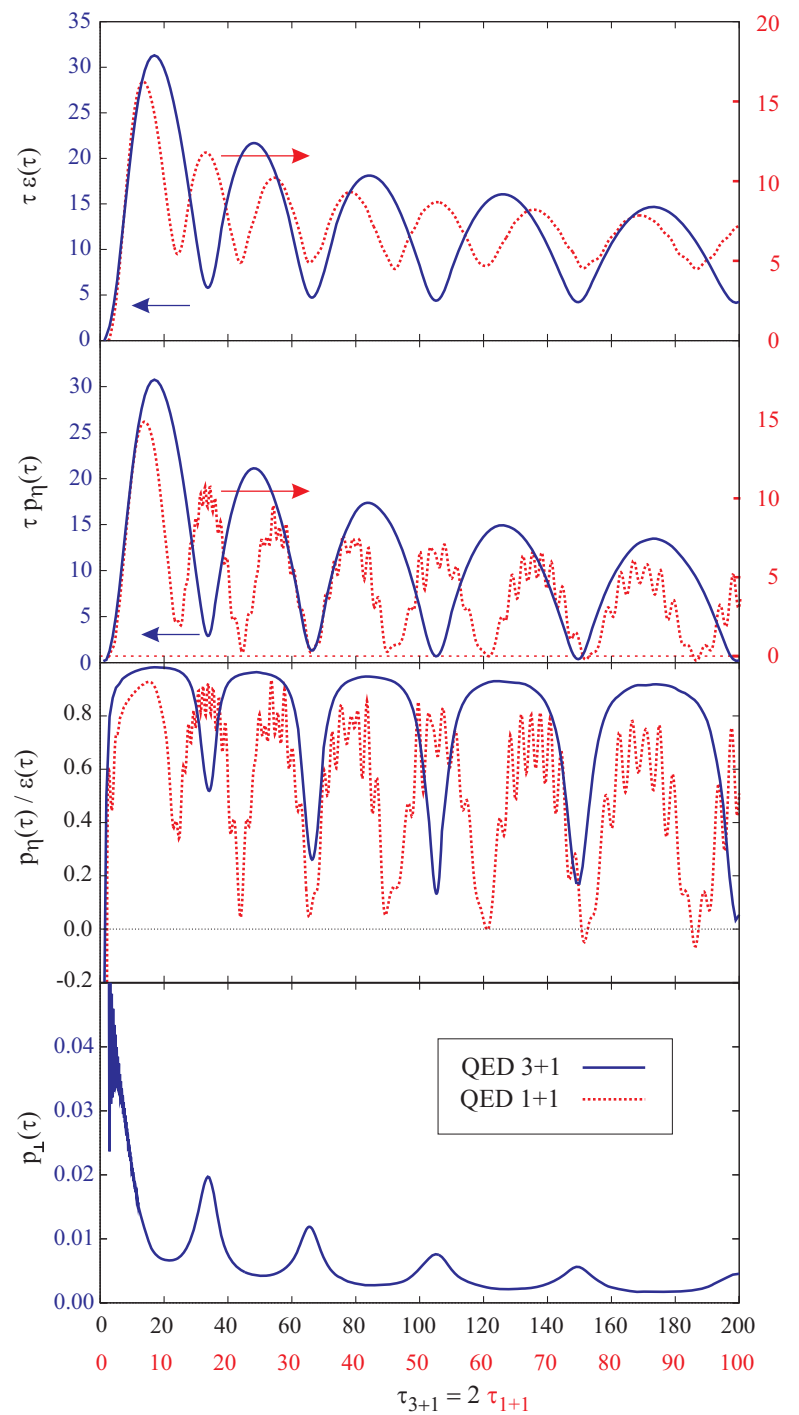

FIG. 5: (Color online) Proper-time evolution of the matter components of the renormalized energy-momentum tensor for the case of (1+1)- and (3+1)-dimensional QED, respectively.

Then we obtain

$$
\begin{aligned}
k_{\tau} & =M \frac{\mathrm{d} \tau}{\mathrm{d} s}=M\left[\left(\frac{\partial \tau}{\partial t}\right)_{z} \frac{\mathrm{d} t}{\mathrm{~d} s}+\left(\frac{\partial \tau}{\partial z}\right)_{t} \frac{\mathrm{d} z}{\mathrm{~d} s}\right] \\
& =\frac{1}{\tau}\left(E t-k_{z} z\right)=\hat{k}^{a} u_{a}=M_{\perp} \cosh (\eta-y),
\end{aligned}
$$

and

$$
\begin{aligned}
k_{\eta} & =M \frac{\mathrm{d} \eta}{\mathrm{d} s}=M\left[\left(\frac{\partial \eta}{\partial t}\right)_{z} \frac{\mathrm{d} t}{\mathrm{~d} s}+\left(\frac{\partial \eta}{\partial z}\right)_{t} \frac{\mathrm{d} z}{\mathrm{~d} s}\right] \\
& =-E z+k_{z} t=-\tau M_{\perp} \sinh (\eta-y) .
\end{aligned}
$$

It follows that $k_{\tau}=\hat{k}^{\mu} u_{\mu}=M_{\perp} \cosh (\eta-y)$ has the meaning of the energy of the particle in the comoving frame.

The interpolating phase-space density $f$ of particles depends on $\left(\tau, k_{\perp}, k_{\eta}\right)$ and is found to be $\eta$-independent. In 


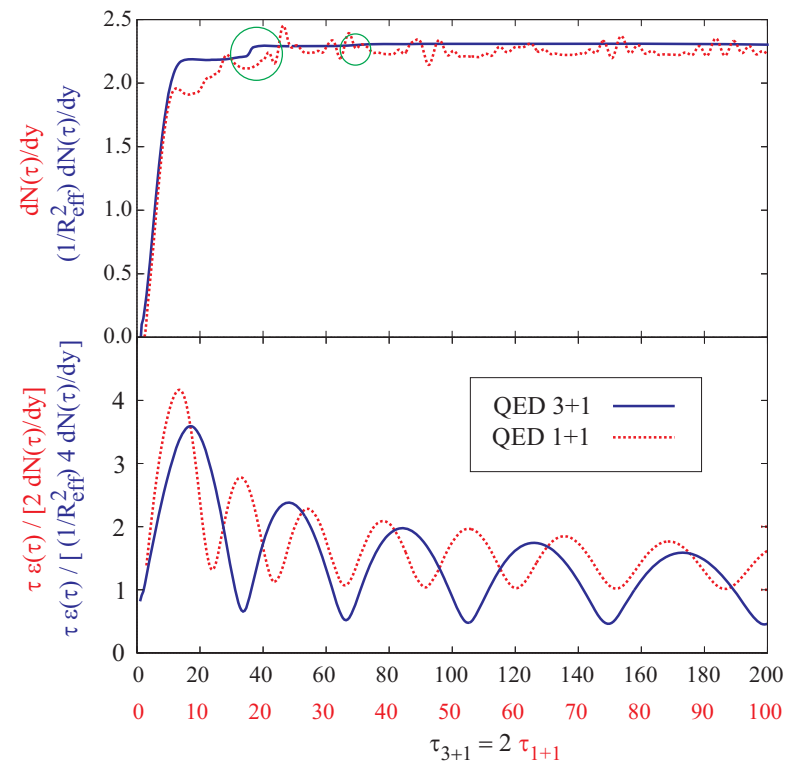

FIG. 6: (Color online) Proper-time evolution of the density of pairs, $\mathrm{d} N / \mathrm{d} y$, and proper time evolution of the ratio $\tau \varepsilon(\tau) /[\mathrm{d} N / \mathrm{d} y]$ for the case of $(1+1)$ - and $(3+1)$-dimensional QED, respectively. The circles in the upper panel denote step increases in the particle density corresponding to the second and third gradients of the current.

order to obtain the center-of-mass particle rapidity and transverse momentum distribution, we change variables from $\left(\eta, k_{\eta}\right)$ to $(z, y)$ at a fixed proper time $\tau$, i.e.

$$
\frac{\mathrm{d}^{6} N}{\mathrm{~d}^{2} x_{\perp} \mathrm{d}^{2} k_{\perp} \mathrm{d} z \mathrm{~d} y}=\frac{\mathrm{d}^{6} N}{\mathrm{~d}^{2} x_{\perp} \mathrm{d}^{2} k_{\perp} \mathrm{d} \eta \mathrm{d} k_{\eta}}\left|\frac{\partial\left(\eta, k_{\eta}\right)}{\partial(z, y)}\right|_{\tau} .
$$

So, from 4.8, we have

$$
\frac{\mathrm{d}^{3} N}{\mathrm{~d}^{2} k_{\perp} \mathrm{d} y}=\iint \mathrm{d}^{2} x_{\perp} \mathrm{d} z\left|\frac{\partial\left(\eta, k_{\eta}\right)}{\partial(z, y)}\right|_{\tau} \frac{f\left(\tau, k_{\perp}, k_{\eta}\right)}{(2 \pi)^{3}},
$$

where the Jacobian is evaluated at a fixed proper time $\tau$,

$$
\begin{aligned}
\left|\frac{\partial\left(\eta, k_{\eta}\right)}{\partial(z, y)}\right|_{\tau} & =\left|\begin{array}{cc}
\partial k_{\eta} / \partial y & \partial k_{\eta} / \partial z \\
\partial \eta / \partial y & \partial \eta / \partial z
\end{array}\right|_{\tau}=\left|\frac{\partial k_{\eta}}{\partial y} \frac{\partial \eta}{\partial z}\right|_{\tau} \\
& =\frac{M_{\perp} \cosh (\eta-y)}{\cosh \eta} .
\end{aligned}
$$

However, since at fixed $\tau$, we have

$$
\left|\frac{\partial k_{\eta}}{\partial y}\right|_{\tau}=\left|\frac{\partial k_{\eta}}{\partial \eta}\right|_{\tau}
$$

we obtain

$$
\left|\frac{\partial\left(\eta, k_{\eta}\right)}{\partial(z, y)}\right|_{\tau}=\left.\frac{\partial k_{\eta}}{\partial z}\right|_{\tau}
$$

Calling the integration over the transverse dimensions the effective transverse size of the colliding ions $A_{\perp}=\pi R_{\text {eff }}^{2}$ we then find from 4.18 that:

$$
\frac{\mathrm{d}^{3} N}{\mathrm{~d}^{2} k_{\perp} \mathrm{d} y}=\frac{A_{\perp}}{(2 \pi)^{3}} \int \mathrm{d} k_{\eta} f\left(\tau, k_{\perp}, k_{\eta}\right) \equiv \frac{\mathrm{d}^{3} N}{\mathrm{~d}^{2} k_{\perp} \mathrm{d} \eta} .
$$

The quantity in Eq. 4.22 is independent of $y$ which is a consequence of the assumed boost invariance. Therefore, using the property of the Jacobean, we have proven that the distribution of particles in particle rapidity is the same as the distribution of particles in fluid rapidity, verifying that in the boost-invariant regime Landau's intuition was correct 29.

We now want to motivate the Cooper-Frye formula used to calculate particle spectrum in hydrodynamical models of particle production [27. We have that a constant $\tau$ surface, which is the freeze-out surface of Landau, is parametrized as

$$
\begin{aligned}
\mathrm{d} \Sigma^{a} & =A_{\perp}(\mathrm{d} z, 0,0, \mathrm{~d} t) \\
& =A_{\perp} \tau \mathrm{d} \eta(\cosh \eta, 0,0, \sinh \eta) .
\end{aligned}
$$

Therefore, we find

$$
\begin{aligned}
\hat{k}^{a} \mathrm{~d} \Sigma_{a} & =A_{\perp} M_{\perp} \tau \cosh (\eta-y) \mathrm{d} \eta \\
& =A_{\perp}\left|\mathrm{d} k_{\eta}\right| .
\end{aligned}
$$

Thus, we can rewrite our expression for the field theory particle spectra as

$$
\begin{aligned}
\frac{\mathrm{d}^{3} N}{\mathrm{~d}^{2} k_{\perp} \mathrm{d} y} & =\frac{A_{\perp}}{(2 \pi)^{3}} \int \mathrm{d} k_{\eta} f\left(\tau, k_{\perp}, k_{\eta}\right) \\
& =\int \hat{k}^{a} \mathrm{~d} \Sigma_{a} f\left(\tau, k_{\perp}, k_{\eta}\right),
\end{aligned}
$$

where in the integration we keep $y$ and $\tau$ fixed. Thus, with the replacement of the thermal single-particle distribution by the quasiparticle distribution function, we get via the coordinate transformation to the center-ofmass frame the Cooper-Frye formula. For completeness, we note that in $(1+1)$ dimensions the particle spectra, $\mathrm{d} N / \mathrm{d} y$, are given by the integral in Eq. 4.10.

The boost invariant assumption leads to an energy momentum tensor which is diagonal in the $(\tau, \rho, \theta, \eta)$ coordinate system which is thus a comoving one. In that system one has for the matter energy-momentum tensor

$$
T_{\mu \nu}=\operatorname{diag}\left(\mathcal{E}, \mathcal{P}_{\rho}, \rho^{2} \mathcal{P}_{\theta}, \tau^{2} \mathcal{P}_{\eta}\right) .
$$

Thus we find in this approximation that there are two separate pressures, one in the longitudinal direction and one in the transverse direction which is quite different from the thermal equilibrium case. However only the longitudinal pressure enters into the energy conservation equation:

$$
\frac{\mathrm{d}(\tau \mathcal{E})}{\mathrm{d} \tau}+\mathcal{P}_{\eta}=E J_{\eta}
$$

It is useful to rewrite the conservation of energy in the out regime as :

$$
\frac{\mathrm{d} \mathcal{E}}{\mathrm{d} \tau}+\frac{\mathcal{E}+\mathcal{P}_{\eta}}{\tau}=0
$$


So to the extent that the ultra-relativistic onedimensional equation of state $\mathcal{E}=\mathcal{P}_{\eta}$ is true, then one has the simple result

$$
\mathcal{E} \propto \tau^{-2}
$$

It turns out, as our numerical results show below, that although $p_{\eta} / \varepsilon \approx 1$ for part of the period of the oscillation, during the minima $p_{\eta} \rightarrow 0$ and this seems to be governing the falloff which is more like $\varepsilon \propto 1 / \tau$.

\section{NUMERICAL RESULTS}

Let us review the equations we intend to solve numerically. The first of these is the polarization equation 2.39

$$
\partial_{\tau} \mathbf{P}_{k}^{(\lambda)}(\tau)=2 \mathbf{k}_{k}(\tau) \times \mathbf{P}_{k}^{(\lambda)}(\tau)
$$

and the second is the backreaction equation 2.71

$$
\begin{gathered}
\partial_{\tau} E(\tau)=\frac{e}{1+e^{2}\left(\delta e^{2}\right)} \sum_{p}^{\Lambda}\left[P_{1 ; k_{\perp}, h}^{(+)}\left(\pi_{\eta}, \tau\right)\right. \\
\left.-\frac{\pi_{\eta}}{\omega}+\frac{e E(\tau)}{\tau}\left(k_{\perp}^{2}+M^{2}\right)\left(\frac{1}{4 \omega^{5}}-\frac{5 \pi_{\eta}^{2}}{4 \omega^{7}}\right)\right] .
\end{gathered}
$$

Here we have subtracted from the integral the adiabatic expansion of $P_{1 ; k_{\perp}, h}^{(+)}\left(\pi_{\eta}, \tau\right)$.

In order to solve the coupled Dirac and backreaction equations, we construct a grid in $k_{\perp}$ and $k_{\eta}$ space as follows: The $k_{\eta}$-momentum variable is discretized on a nonuniform piece-wise momentum grid with a cutoff at $k_{\eta}=\Lambda_{\eta}$; we find that a value of $\Lambda_{k} \approx 500$ is necessary to obtain numerical results insensitive with respect to the cutoff. A similar nonuniform grid is used to discretize the $k_{\perp}$ variable, $k_{\perp} \in[0, \Lambda]$. A fourth-order Runge-Kutta method is employed to solve the coupled Dirac equation and backreaction problem.

For the purpose of calculating the subtracted values of the current $J(\tau)$ and the components of the matter energy-momentum tensor, we compute the momentum integrals symmetrically with respect to the variable $\pi_{\eta}$ rather than $k_{\eta}$. The corresponding momentum cutoff in $\pi_{\eta}$-space is chosen to be $20 \%$ greater than $\tau_{\max } \Lambda_{\eta}$ to allow for possible very large values of $A(\tau)$, the latter being unknown at the beginning of the calculation.

The momentum integrals with respect to $\pi_{\eta}$ and $k_{\perp}$ are performed using a Chebyshev integration method with spectral convergence 31]. Using this procedure, we found that a grid of approximately 8000 points in the $k_{\eta}$ (or $\pi_{\eta}$ ) variable and 128 grid points in the $k_{\perp}$ variable is necessary to obtain a converged numerical result. As such, the calculations for the backreaction problem in (3+1)-dimensional QED require at least 100 times larger storage and computational time then the corresponding (1+1)-dimensional QED problem.

For illustrative purposes, we took: $m=1, e=1$, $\tau_{0}=1 / m=1, A\left(\tau_{0}\right)=0$, and $E\left(\tau_{0}\right)=4$. These strong-field initial conditions have been shown to produce sufficient fermion pairs at $\tau=\tau_{0}$ for plasma oscillations to take place. Just like in the $(1+1)$-dimensional case, the conservation of the energy-momentum tensor, see Eq. (3.25), serves as a numerical test: for the results of simulations reported here, the renormalized energymomentum tensor is conserved within machine precision.

In order to keep the size of the simulation to a minimum, we chose the initial conditions corresponding to the one-field scenario introduced first in Ref. 24 and summarized in Sec. IIC. In Figs. 1 we illustrate the convergence of our results with respect to the choice of the cutoff, $\Lambda$. For completeness, we depict the proper-time evolution of the fields, $A(\tau)$ and $E(\tau)$, current, $J(\tau)$, energy, $\epsilon(\tau)$ and transverse pressure, $p_{\eta}(\tau)$, for cutoff values between 2 and 6 . We conclude that for $\Lambda=5$ the results are insensitive to the cutoff $\Lambda$, within numerical accuracy.

It is important to note that our choice of initial conditions results in a time evolution that is not consistent with the adiabatic expansion for early values of the proper time. In Fig. 2 we depict the proper-time evolution of the fields, $A(\tau)$ and $E(\tau)$, and current, $J(\tau)$, at early times for several values of the cutoff $\Lambda$. We note that while the proper-time dynamics converges for $\tau>5$, for earlier times the proper-time evolution depends on the choice of the cutoff $\Lambda$. However, for larger proper-time values, the nonadiabatic components of the current dissipate and the adiabatic-expansion-based subtraction becomes exact. This behavior is illustrated numerically in Fig. 3. where we depict the $k_{\perp}$-projected distribution of the current for $\tau$ values of 2,3 and 9 . We notice that the nonadiabatic oscillations of the current present at early proper times dampen out and disappear at later proper times. Therefore, the early proper-time evolution will be disregarded as "unphysical." This is a small price to pay in order to keep the storage and time requirements of our simulation to a minimum.

In Fig. 4, we compare the proper-time evolution of the electromagnetic field, $A(\tau)$, electric field, $E(\tau)$, and current, $J(\tau)$, for $(1+1)$ - and $(3+1)$-dimensional QED, respectively. Similarly, in Fig. 55 we depict the propertime evolution of the matter components of the energymomentum tensor. We note that in $(1+1)$ dimensions the fields evolve much faster than in $(3+1)$ dimensions. Also, in $(3+1)$-dimensional QED the energy and longitudinalpressure densities are very very close in magnitude, which in turn results in a small transverse pressure, $p_{\perp}(\tau)$. Qualitatively, we also note that the modulation observed in the proper-time evolution of the current and longitudinal pressure in $(1+1)$-dimensional QED are not present any longer in $(3+1)$ dimensions. Also by inspecting the two upper panels in Fig. 5, we notice that in $3+1$ dimensions $p_{\eta} \approx \varepsilon$. However, the ratio $p_{\eta} / \varepsilon$ becomes close to zero near the minimum of the oscillation and this in turn leads to $\tau \varepsilon$ to be almost constant instead of going as $1 / \tau$ using the arguments coming from the energy conservation equation Eq. 4.28.

Finally, the proper-time evolution of the density of 


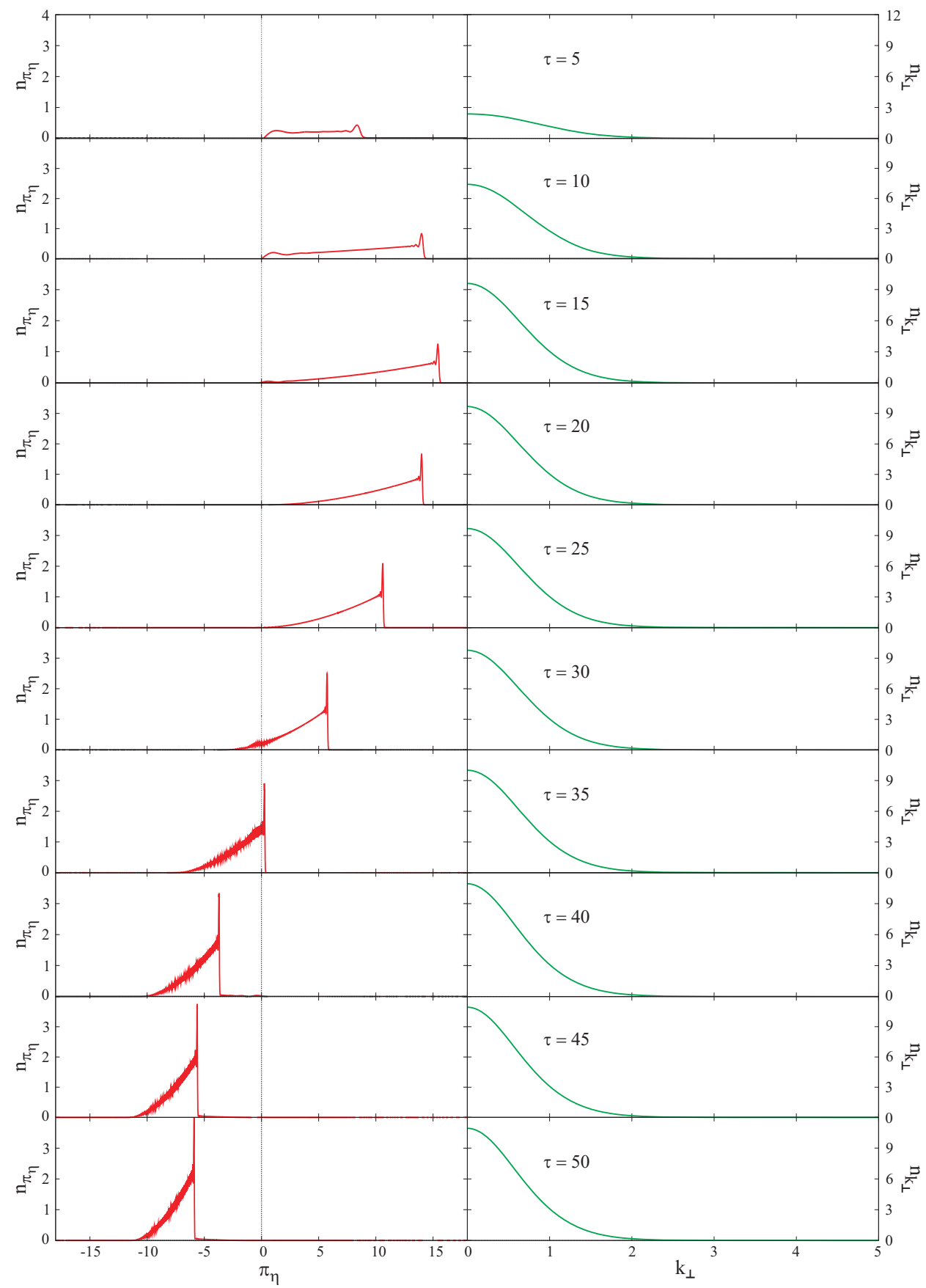

FIG. 7: (Color online) Proper-time evolution of the longitudinal momentum-dependent pair-density distribution, $n_{\pi_{\eta}}$, and the proper-time evolution of the transverse momentum-dependent pair-density distribution, $n_{k_{\perp}}$. (See also Ref. 30)

pairs, $\mathrm{d} N / \mathrm{d} y$, are depicted in Fig. 6. Particles are being created corresponding to the current gradients, with the major contribution corresponding to the initial current gradient, and subsequent smaller step increases before the particle density saturates. At late values of the proper time, the ratios $\tau \varepsilon(\tau) /[\mathrm{d} N / \mathrm{d} y]$ are seen to approach a constant consistent with the hydrodynamical picture, which relates the energy in a bin of rapidity divided by the energy of a single particle with that rapidity with the number of particles in a bin of rapidity as explained in Ref. 23. In the real problem we expect that interactions between the fermions will eliminate the oscillations observed here.

The proper-time evolution of the momentumdependent longitudinal pair-density distribution, $n_{\pi_{\eta}}$, defined as

$$
n_{\pi_{\eta}}=\frac{2 \pi}{A_{\perp}} \frac{\mathrm{d}^{2} N}{\mathrm{~d} k_{\eta} \mathrm{d} y}=\int_{0}^{+\infty} \frac{k_{\perp} \mathrm{d} k_{\perp}}{2 \pi} f\left(\tau, k_{\perp}, k_{\eta}\right),
$$




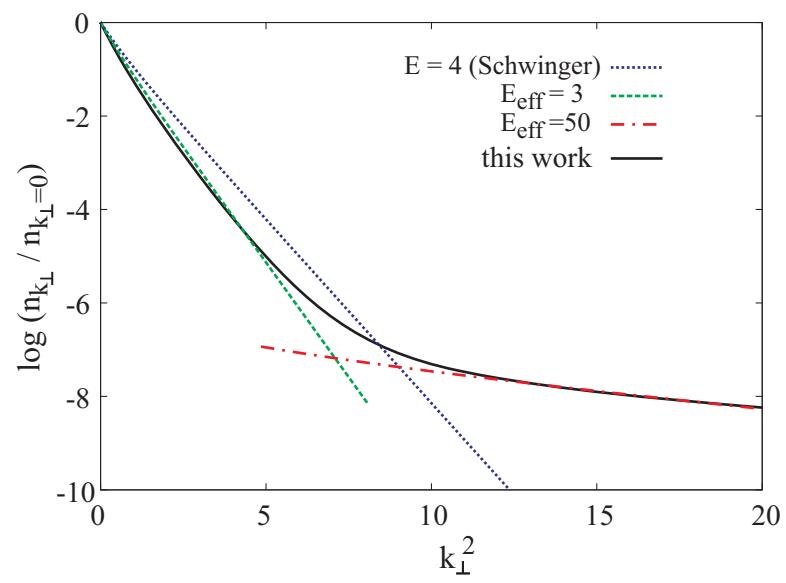

FIG. 8: (Color online) Comparison of the transverse distribution of particles according to our numerical simulation and the transverse distribution of particles for the constant electromagnetic field case with $e E=4$ as described by Eq. 11.2.

and the transverse pair-density distribution, $n_{k_{\perp}}$, defined as

$$
n_{k_{\perp}}=\frac{(2 \pi)^{2}}{A_{\perp}} \frac{\mathrm{d}^{3} N}{\mathrm{~d}^{2} k_{\perp} \mathrm{d} y}=\int_{-\infty}^{+\infty} \frac{\mathrm{d} k_{\eta}}{2 \pi} f\left(\tau, k_{\perp}, k_{\eta}\right),
$$

in (3+1)-dimensional QED are shown in Fig. 7. (See also Ref. [30.) We note that the centroid of the particledensity distribution, $n_{\pi_{\eta}}$, oscillates between positive and negative values of $\pi_{\eta}$, similar to the $(1+1)$-dimensional QED case.

In Fig. 8 we compare the transverse momentum distribution given by the constant field exact solution Eq. 11.2 with the results of our numerical solution for $e E=4$. Part of the results are expected, that is at small transverse momenta the distribution of particles is similar to the static case but with a smaller effective field since the field is decreasing during the first phase of particle production. What is unexpected is that, in the problem with backreaction, there is a new tail in the transverse momentum distribution which falls exponentially with an effective $|e E|=50$. This is a totally surprising result whose origin we do not yet have a simple explanation for.

\section{CONCLUSIONS}

We have for the first time calculated the transverse distribution of jets produced by an initial strong electric field including the effects of backreaction. We have compared the results of our $(3+1)$-dimensional calculations (for "hydrodynamic" quantities as well as for the proper time evolution of the electric field and current) with their $(1+1)$-dimensional counterparts. We find that the electric field degrades much quicker in $(3+1)$ dimensions than in $(1+1)$ dimensions. Also secondary oscillations in the current and in the longitudinal pressure, present in $(1+1)$ dimensions seem to be absent in $(3+1)$ dimensions suggesting that the extra degrees of freedom perform some smoothing. We now have the first numerical results for the transverse momentum distribution function of fermion pairs which we can compare with the exact results for the constant field problem. We find that unlike the constant field case, the distribution is bimodal. At modest $k_{\perp}^{2} \leq 5 m^{2}$ the transverse distribution is similar to the constant field case with a reduced $(75 \%)$ effective $e E$ for $e E_{0}=4$. For larger transverse momentum $k_{\perp}^{2} \geq 10 \mathrm{~m}^{2}$ the transverse distribution function has a tail described by an effective $e E$ which is of the order of 50. This is a totally new feature that is as of yet not understood simply. In a related paper [32] we will also consider a transport approach to the $(3+1)$-dimensional problem and show that such a semiclassical picture works better in $(3+1)$ than in $(1+1)$ dimensions.

\section{Acknowledgments}

This work was performed in part under the auspices of the United States Department of Energy. The authors would like to thank the Santa Fe Institute for its hospitality during the completion of this work.

\section{APPENDIX A: NOTATION}

In this appendix, we list our notation and conventions used throughout this paper. We use a boldface $\mathbf{k}$ to designate the complete set of mode variables, $k$ and $k_{m}$ subsets of the full set, and $k_{\phi}$ the cylindrical coordinate set. In addition, the set $p$ substitutes the $\pi_{\eta}$ kinetic momentum for $k_{\eta}$. These sets are given by

$$
\begin{aligned}
\mathbf{k} & \equiv\left(k_{\perp}, h, k_{\eta}, m\right), \\
k & \equiv\left(k_{\perp}, h, k_{\eta}\right), \\
p & \equiv\left(k_{\perp}, h, \pi_{\eta}\right), \\
k_{m} & \equiv\left(k_{\perp}, m\right), \\
k_{\phi} & \equiv\left(k_{x}, k_{y}, k_{\eta}\right)=\left(k_{\perp}, \phi, k_{\eta}\right) .
\end{aligned}
$$

Sums over these quantities indicate the following integrals and sums

$$
\begin{aligned}
\sum_{\mathbf{k}} & \equiv \int_{0}^{+\infty} \frac{k_{\perp} \mathrm{d} k_{\perp}}{2 \pi} \int_{-\infty}^{+\infty} \frac{\mathrm{d} k_{\eta}}{2 \pi} \sum_{m=-\infty}^{+\infty} \sum_{h= \pm 1}, \\
\sum_{k} & \equiv \int_{0}^{+\infty} \frac{k_{\perp} \mathrm{d} k_{\perp}}{2 \pi} \int_{-\infty}^{+\infty} \frac{\mathrm{d} k_{\eta}}{2 \pi} \sum_{h= \pm 1} \\
\sum_{p} & \equiv \int_{0}^{+\infty} \frac{k_{\perp} \mathrm{d} k_{\perp}}{2 \pi} \int_{-\infty}^{+\infty} \frac{\mathrm{d} \pi_{\eta}}{2 \pi} \sum_{h= \pm 1}, \\
\sum_{k_{\phi}} & \equiv \int_{-\infty}^{+\infty} \frac{\mathrm{d} k_{x}}{2 \pi} \int_{-\infty}^{+\infty} \frac{\mathrm{d} k_{y}}{2 \pi} \int_{-\infty}^{+\infty} \frac{\mathrm{d} k_{\eta}}{2 \pi} \\
& =\int_{0}^{\infty} \frac{k_{\perp} \mathrm{d} k_{\perp}}{2 \pi} \int_{0}^{2 \pi} \frac{\mathrm{d} \phi}{2 \pi} \int_{-\infty}^{+\infty} \frac{\mathrm{d} k_{\eta}}{2 \pi}
\end{aligned}
$$


We also use the following notation for $\delta$-functions

$$
\delta_{\mathbf{k}, \mathbf{k}^{\prime}} \equiv(2 \pi)^{2} \delta_{h, h^{\prime}} \delta_{m, m^{\prime}} \frac{\delta\left(k_{\perp}-k_{\perp}^{\prime}\right)}{\sqrt{k_{\perp} k_{\perp}^{\prime}}} \delta\left(k_{\eta}-k_{\eta}^{\prime}\right) .
$$

In a similar way, we put $\mathbf{x} \equiv(\rho, \theta, \eta)$. The sum over $\mathbf{x}$ means

$$
\sum_{\mathbf{x}} \equiv \int_{0}^{\infty} \frac{\rho \mathrm{d} \rho}{2 \pi} \int_{0}^{2 \pi} \frac{\mathrm{d} \theta}{2 \pi} \int_{-\infty}^{+\infty} \frac{\mathrm{d} \eta}{2 \pi}
$$

and $\delta_{\mathbf{x}, \mathbf{x}^{\prime}}$ means

$$
\delta_{\mathbf{x}, \mathbf{x}^{\prime}} \equiv \frac{\delta\left(\rho-\rho^{\prime}\right)}{\sqrt{\rho \rho^{\prime}}} \delta\left(\theta-\theta^{\prime}\right) \delta\left(\eta-\eta^{\prime}\right) .
$$

\section{APPENDIX B: TRANSVERSE HELICITY EIGENVECTORS}

In this section we derive transverse helicity eigenvectors and show how they can be used to expand solutions of the Dirac equation in boost-invariant coordinates. The Hermitian two-component transverse helicity operator $H_{\perp}$ is defined in momentum space by

$$
H_{\perp}=\frac{1}{k_{\perp}}(\mathbf{k} \times \boldsymbol{\sigma}) \cdot \hat{\mathbf{e}}_{z}=\left(\begin{array}{cc}
0 & -i e^{-i \phi} \\
+i e^{+i \phi} & 0
\end{array}\right),
$$

where $k_{x}=k_{\perp} \cos \phi$ and $k_{y}=k_{\perp} \sin \phi$. We write the eigenvalue equation for this operator as:

$$
H_{\perp} \chi_{\phi, h}=h \chi_{\phi, h},
$$

with eigenvalues $h= \pm 1$ and orthogonal eigenvectors:

$$
\chi_{\phi, h}=\frac{1}{\sqrt{2}}\left(\begin{array}{c}
1 \\
i h e^{i \phi}
\end{array}\right) .
$$

We note that $\sigma_{z} \chi_{\phi, h}=\chi_{\phi,-h}$, and that:

$$
\left(\sigma_{x} k_{x}+\sigma_{y} k_{y}\right) \chi_{\phi, h}=i h k_{\perp} \chi_{\phi,-h} .
$$

For the coordinate system $x^{\mu}=(\tau, x, y, \eta)$, Dirac's equation is:

$$
\left\{\tilde{\gamma}^{\mu}(\tau, \eta)\left[i \partial_{\mu}-g A_{\mu}(\tau)\right]-M\right\} \psi(\tau, x, y, \eta)=0,
$$

where now:

$$
\begin{aligned}
\tilde{\gamma}^{\tau}(\eta) & =\cosh \eta \gamma^{0}-\sinh \eta \gamma^{3} \\
\tilde{\gamma}^{\eta}(\tau, \eta) & =\left(-\sinh \eta \gamma^{0}+\cosh \eta \gamma^{3}\right) / \tau .
\end{aligned}
$$

with $\tilde{\gamma}^{x}=\gamma^{1}$ and $\tilde{\gamma}^{y}=\gamma^{2}$. We next boost to a coordinate system where $\eta=0$ by setting:

$$
\psi(\tau, x, y, \eta)=S(\eta) \phi^{\prime}(\tau, x, y, \eta) / \sqrt{\tau} .
$$

then $(\mathrm{B} 5)$ becomes

$$
\begin{aligned}
& \left\{i \gamma^{0} \partial_{\tau}+i \gamma^{1} \partial_{x}+i \gamma^{2} \partial_{y}\right. \\
& \left.+\gamma^{3}\left[i \partial_{\eta}+g A(\tau)\right] / \tau-M\right\} \phi^{\prime}(\tau, x, y, \eta)=0,
\end{aligned}
$$

which is what we want to solve. So let us first introduce the Fourier transform:

$$
\phi^{\prime}(\tau, x, y, \eta)=\phi_{\mathbf{k}}^{\prime}(\tau) e^{i\left(k_{x} x+k_{y} y+k_{\eta} \eta\right)},
$$

where $\mathbf{k}=\left(k_{\eta}, k_{x}, k_{y}\right)$. Then (B7) becomes:

$$
\left[i \gamma^{0} \partial_{\tau}-\gamma^{1} k_{x}-\gamma^{2} k_{y}-\gamma^{3} \pi_{\eta}(\tau)-M\right] \phi_{k}^{\prime}(\tau)=0,
$$

where $\pi_{\eta}(\tau)=\left[k_{\eta}-e A(\tau)\right] / \tau$ is the kinetic momentum. Using Eq. (B4), we see that $(\bar{B} 9)$ is separable if we put

$$
\phi_{k}^{\prime}(\tau)=\left(\begin{array}{c}
\phi_{(+) ; k}(\tau) \chi_{\phi,+h} \\
\phi_{(-) ; k}(\tau) \chi_{\phi,-h}
\end{array}\right),
$$

where $\phi_{( \pm) ; k}(\tau)$ now satisfy the two-component equation

$$
\begin{aligned}
i \partial_{\tau} & \left(\begin{array}{l}
\phi_{(+) ; k}(\tau) \\
\phi_{(-) ; k}(\tau)
\end{array}\right) \\
\quad & \left(\begin{array}{cc}
+M & \pi_{\eta}(\tau)-i h k_{\perp} \\
\pi_{\eta}(\tau)+i h k_{\perp} & -M
\end{array}\right)\left(\begin{array}{l}
\phi_{(+) ; k}(\tau) \\
\phi_{(-) ; k}(\tau)
\end{array}\right),
\end{aligned}
$$

which agrees with Eq. 2.35). Near $\tau=\tau_{0}$, there are positive and negative energy solutions to these equations which we label by $\lambda= \pm 1$. So the fermi field $\hat{\phi}(\tau, x, y, \eta)$ can be expanded as

$$
\begin{aligned}
\hat{\phi}^{\prime}(\tau, x, y, \eta) & =\sum_{k_{\phi}} \sum_{h= \pm 1} \sum_{\lambda= \pm 1} \hat{A}_{k_{\phi}, h}^{(\lambda)} \\
& \times\left(\begin{array}{l}
\phi_{(+) ; k}^{(\lambda)}(\tau) \chi_{\phi,+h} \\
\phi_{(-) ; k}^{(\lambda)}(\tau) \chi_{\phi,-h}
\end{array}\right) e^{i\left(k_{\eta} \eta+k_{x} x+k_{y} y\right)}
\end{aligned}
$$

where $\hat{A}_{k_{\phi}, h}^{(\lambda)}$ are the creation and annihilation operators for the state described by $\left(k_{\phi}, h, \lambda\right)$. Now let us introduce cylindrical coordinates, $x=\rho \cos \theta$ and $y=\rho \sin \theta$, so that

$$
k_{x} x+k_{y} y=k_{\perp} \rho \cos (\theta-\phi) .
$$

Now the generating function for Bessel functions is given by

$$
\exp [z(t-1 / t) / 2]=\sum_{m=-\infty}^{+\infty} t^{m} J_{m}(z) .
$$

If we put $t=i e^{i(\theta-\phi)}$ and $z=k_{\perp} \rho$, this becomes

$$
\exp \left[i k_{\perp} \rho \cos (\theta-\phi)\right]=\sum_{m=-\infty}^{+\infty} i^{m} e^{i m(\theta-\phi)} J_{m}\left(k_{\perp} \rho\right) .
$$

Using these results in Eq. (B11), we find in cylindrical coordinates the expansion,

$$
\begin{gathered}
\hat{\phi}^{\prime}(\tau, \rho, \theta, \eta)=\sum_{k_{\phi}} \sum_{m=-\infty}^{+\infty} \sum_{h= \pm 1} \sum_{\lambda= \pm 1} \hat{A}_{k_{\phi}, h}^{(\lambda)} e^{i k_{\eta} \eta} \\
\times\left(\begin{array}{c}
\phi_{(+) ; k}^{(\lambda)}(\tau) \chi_{\phi,+h} \\
\phi_{(-) ; k}^{(\lambda)}(\tau) \chi_{\phi,-h}
\end{array}\right) i^{m} e^{i m(\theta-\phi)} J_{m}\left(k_{\perp} \rho\right) .
\end{gathered}
$$


Now let us define the Fourier transform pair:

$$
\begin{aligned}
i^{m} \hat{A}_{k_{\eta}, k_{\perp}, m, h}^{(\lambda)} & =\int_{0}^{2 \pi} \frac{\mathrm{d} \phi}{2 \pi} \hat{A}_{k_{\eta}, k_{\perp}, \phi, h}^{(\lambda)} e^{-i m \phi}, \\
\hat{A}_{k_{\eta}, k_{\perp}, \phi, h}^{(\lambda)} & =\sum_{m=-\infty}^{+\infty} i^{m} \hat{A}_{k_{\eta}, k_{\perp}, m, h}^{(\lambda)} e^{i m \phi} .
\end{aligned}
$$

So using (B15a), and putting $m \rightarrow m+1$ in the second and fourth components, Eq. (B14) becomes

$$
\begin{aligned}
\hat{\phi}^{\prime}(\tau, \rho, \theta, \eta) & =\sum_{\mathbf{k}} \sum_{\lambda= \pm 1} \hat{A}_{\mathbf{k}}^{(\lambda)} \\
& \times e^{i k_{\eta} \eta}\left(\begin{array}{c}
\phi_{(+) ; k}^{(\lambda)}(\tau) \chi_{k_{m},+h}^{\prime}(\rho, \theta) \\
\phi_{(-) ; k}^{(\lambda)}(\tau) \chi_{k_{m},-h}^{\prime}(\rho, \theta)
\end{array}\right)
\end{aligned}
$$

where now

$$
\chi_{k_{m}, h}^{\prime}(\rho, \theta)=\frac{1}{\sqrt{2}}\left(\begin{array}{c}
e^{i m \theta} J_{m}\left(k_{\perp} \rho\right) \\
-h e^{i(m+1) \theta} J_{m+1}\left(k_{\perp} \rho\right)
\end{array}\right) .
$$

Finally, we boost to a coordinate system where $\theta=0$ by multiplying $\hat{\phi}^{\prime}(\tau, \rho, \theta, \eta)$ by $S_{\rho}^{-1}(\theta)$, which can be written as

$$
\begin{aligned}
S_{\rho}^{-1}(\theta) & =\cos (\theta / 2)-\gamma^{1} \gamma^{2} \sin (\theta / 2) \\
& =\left(\begin{array}{cccc}
e^{+i \theta / 2} & 0 & 0 & 0 \\
0 & e^{-i \theta / 2} & 0 & 0 \\
0 & 0 & e^{+i \theta / 2} & 0 \\
0 & 0 & 0 & e^{-i \theta / 2}
\end{array}\right)
\end{aligned}
$$

so that $\hat{\psi}(x)=S(\theta, \eta) \hat{\phi}(x)$ and from Eq. B16, we find

$$
\hat{\phi}(x)=S_{\rho}^{-1}(\theta) \hat{\phi}^{\prime}(x)=\sum_{\mathbf{k}} \sum_{\lambda= \pm 1} \hat{A}_{\mathbf{k}}^{(\lambda)} \phi_{\mathbf{k}}^{(\lambda)}(x),
$$

where

$$
\phi_{\mathbf{k}}^{(\lambda)}(x) \equiv e^{i k_{\eta} \eta}\left(\begin{array}{c}
\phi_{(+) ; k}^{(\lambda)}(\tau) \chi_{k_{m},+h}(\rho, \theta) \\
\phi_{(-) ; k}^{(\lambda)}(\tau) \chi_{k_{m},-h}(\rho, \theta)
\end{array}\right),
$$

and where

$$
\chi_{k_{m}, h}(\rho, \theta)=\frac{e^{i(m+1 / 2) \theta}}{\sqrt{2}}\left(\begin{array}{c}
J_{m}\left(k_{\perp} \rho\right) \\
-h J_{m+1}\left(k_{\perp} \rho\right)
\end{array}\right) .
$$

in agreement with the field expansion given in Eqs. 2.30, 2.32, and 2.33 in Sec. II B. We have shown here that the separation of variables method for the Dirac equation we used in Sec. IIB can easily be understood as an expansion of transverse helicity eigenvectors in boostinvariant coordinates.

The transverse helicity eigenvectors given in Eq. (B21) satisfy the eigenvalue equation,

$$
\begin{aligned}
{\left[i \sigma_{y}\left(\partial_{\rho}+\frac{1}{2 \rho}\right)+i \sigma_{x} \frac{\partial_{\theta}}{\rho}\right] } & \chi_{k_{m}, h}(\rho, \theta) \\
& =h k_{\perp} \chi_{k_{m}, h}(\rho, \theta),
\end{aligned}
$$

are normalized,

$$
\begin{array}{r}
\int_{0}^{\infty} \rho \mathrm{d} \rho \int_{0}^{2 \pi} \mathrm{d} \theta \chi_{k_{m}, h}(\rho, \theta) \chi_{k_{m}^{\prime}, h^{\prime}}(\rho, \theta) \\
=\delta_{h, h^{\prime}} \delta_{m, m^{\prime}} \frac{\delta\left(k_{\perp}-k_{\perp}^{\prime}\right)}{\sqrt{k_{\perp} k_{\perp}^{\prime}}}
\end{array}
$$

and complete

$$
\begin{array}{r}
\int_{0}^{\infty} \frac{k_{\perp} \mathrm{d} k_{\perp}}{2 \pi} \sum_{m=-\infty}^{+\infty} \sum_{h= \pm 1} \chi_{k_{m}, h}(\rho, \theta) \chi_{k_{m}, h}^{\dagger}\left(\rho^{\prime}, \theta^{\prime}\right) \\
=\delta\left(\theta-\theta^{\prime}\right) \frac{\delta\left(\rho-\rho^{\prime}\right)}{\sqrt{\rho \rho^{\prime}}}
\end{array}
$$

\section{APPENDIX C: ADIABATIC EXPANSION OF SOLUTIONS OF THE DIRAC EQUATION}

In this section, we find an adiabatic expansion of the positive energy solutions of the Dirac equation for a slowly varying field $A(\tau)$. It is simplest to obtain an adiabatic expansion of the polarization vector $\mathbf{P}_{k}^{(\lambda)}(\tau)$, which we introduced in Sec. IIB. The equation of motion of the polarization vector was given in Eq. 2.39 as

$$
\dot{\mathbf{P}}_{k}^{(\lambda)}(\tau)=2 \mathbf{k}_{k}(\tau) \times \mathbf{P}_{k}^{(\lambda)}(\tau)
$$

where $\mathbf{k}_{k}(\tau)$ is given by

$$
\mathbf{k}_{k}(\tau)=\pi_{\eta}(\tau) \hat{\mathbf{e}}_{1}+h k_{\perp} \hat{\mathbf{e}}_{2}+M \hat{\mathbf{e}}_{3} .
$$

The initial condition at $\tau=\tau_{0}$ is given in Eq. (2.53) as

$$
\mathbf{P}_{k}^{(\lambda)}\left(\tau_{0}\right) \equiv \mathbf{P}_{0 ; k}^{(\lambda)}=\lambda \mathbf{k}_{0 ; k} / \omega_{0 ; k}
$$

For slowly varying values of $\pi_{\eta}(\tau), \mathbf{P}_{k}^{(\lambda)}(\tau)$ simply precesses about the slowly varying value of $\mathbf{k}_{k}(\tau)$. In order to count derivatives with respect to $\tau$, let us put

$$
\partial_{\tau} \mapsto \epsilon \partial_{\tau}
$$

We next expand $\mathbf{P}_{k}^{(\lambda)}(\tau)$ in powers of $\epsilon$ by writing

$$
\begin{aligned}
& \mathbf{P}=\mathbf{P}^{(0)}+\epsilon \mathbf{P}^{(1)}+\epsilon^{2} \mathbf{P}^{(2)}+\cdots, \\
& \dot{\mathbf{P}}=\epsilon \dot{\mathbf{P}}^{(0)}+\epsilon^{2} \dot{\mathbf{P}}^{(1)}+\epsilon^{3} \dot{\mathbf{P}}^{(2)}+\cdots,
\end{aligned}
$$

Here and in the following, we omit momentum and time dependencies and the $(\lambda)$ label. The superscript now counts powers of $\epsilon$ and the dot refers to derivatives with respect to $\tau$. So substitution of (C5) into Eq. (C1) becomes

$$
\begin{aligned}
\epsilon \dot{\mathbf{P}}^{(0)}+ & \epsilon^{2} \dot{\mathbf{P}}^{(1)}+\epsilon^{3} \dot{\mathbf{P}}^{(2)}+\cdots \\
& =2 \mathbf{k} \times\left[\mathbf{P}^{(0)}+\epsilon \mathbf{P}^{(1)}+\epsilon^{2} \mathbf{P}^{(2)}+\cdots\right]
\end{aligned}
$$


Equating equal powers of $\epsilon$ gives

$$
\begin{aligned}
& 2 \mathbf{k} \times \mathbf{P}^{(0)}=0 \\
& 2 \mathbf{k} \times \mathbf{P}^{(1)}=\dot{\mathbf{P}}^{(0)}, \\
& 2 \mathbf{k} \times \mathbf{P}^{(2)}=\dot{\mathbf{P}}^{(1)}, \\
& 2 \mathbf{k} \times \mathbf{P}^{(3)}=\dot{\mathbf{P}}^{(2)}, \quad \text { etc } \cdots
\end{aligned}
$$

Let us introduce transverse and longitudinal components of the polarization vector by writing $\mathbf{P}^{(\kappa)}=\mathbf{P}_{T}^{(\kappa)}+\mathbf{P}_{L}^{(\kappa)}$, where $\mathbf{k} \cdot \mathbf{P}_{T}^{(\kappa)}=0$ and $\mathbf{k} \times \mathbf{P}_{L}^{(\kappa)}=0$. So Eqs. (C7) determine only the transverse components of the polarization vector. The longitudinal portion is then fixed by the normalization requirement, as we will see below.

From Eq. C7a, $\mathbf{P}^{(0)}$ is entirely longitudinal and has the normalized solution

$$
\mathbf{P}^{(0)}=\mathbf{k} / \omega, \quad \omega=|\mathbf{k}|=\sqrt{\pi_{\eta}^{2}+k_{\perp}^{2}+M^{2}} .
$$

So $\mathbf{P}^{(0)}=\mathbf{P}_{0}$. So we have

$$
\dot{\mathbf{P}}^{(0)}=\frac{\dot{\mathbf{k}}}{\omega}-\frac{\mathbf{k} \dot{\omega}}{\omega^{2}}=\frac{\dot{\mathbf{k}}}{\omega}-\frac{\mathbf{k}(\dot{\mathbf{k}} \cdot \mathbf{k})}{\omega^{3}}=\frac{\mathbf{k} \times(\dot{\mathbf{k}} \times \mathbf{k})}{\omega^{3}} .
$$

Then Eq. (C7b) becomes

$$
2 \mathbf{k} \times \mathbf{P}^{(1)}=\frac{\mathbf{k} \times(\dot{\mathbf{k}} \times \mathbf{k})}{\omega^{3}}
$$

so the transverse component of $\mathbf{P}^{(1)}$ is given by

$$
\mathbf{P}_{T}^{(1)}=\frac{\dot{\mathbf{k}} \times \mathbf{k}}{2 \omega^{3}}
$$

We will choose the longitudinal component $\mathbf{P}_{L}^{(1)}=0$, so that

$$
\mathbf{P}^{(1)}=\frac{\dot{\mathbf{k}} \times \mathbf{k}}{2 \omega^{3}}=-\frac{\dot{\pi}_{\eta}}{2 \omega^{3}}\left(M \hat{\mathbf{e}}_{2}-h k_{\perp} \hat{\mathbf{e}}_{3}\right) .
$$

Then to first order, $\mathbf{P}=\mathbf{P}^{(0)}+\epsilon \mathbf{P}^{(1)}$, the polarization vector is normalized to this order, since

$$
P^{2}=P_{0}^{2}+2 \epsilon \mathbf{P}^{(0)} \cdot \mathbf{P}^{(1)}=1 .
$$

From $\mathrm{C} 12$, we find

$$
\begin{aligned}
\dot{\mathbf{P}}^{(1)} & =\frac{\ddot{\mathbf{k}} \times \mathbf{k}}{2 \omega^{3}}-\frac{3(\dot{\mathbf{k}} \times \mathbf{k}) \dot{\omega}}{2 \omega^{4}} \\
& =\frac{\ddot{\mathbf{k}} \times \mathbf{k}}{2 \omega^{3}}-\frac{3(\dot{\mathbf{k}} \times \mathbf{k})(\dot{\mathbf{k}} \cdot \mathbf{k})}{2 \omega^{5}} \\
& =\mathbf{k} \times\left[\frac{3(\dot{\mathbf{k}} \cdot \mathbf{k}) \dot{\mathbf{k}}-\omega^{2} \ddot{\mathbf{k}}}{2 \omega^{5}}\right] .
\end{aligned}
$$

Then Eq. C7c becomes

$$
2 \mathbf{k} \times \mathbf{P}^{(2)}=\mathbf{k} \times\left[\frac{3(\dot{\mathbf{k}} \cdot \mathbf{k}) \dot{\mathbf{k}}-\omega^{2} \ddot{\mathbf{k}}}{2 \omega^{5}}\right],
$$

so adding a longitudinal part to $\mathbf{P}^{(2)}$, we find

$$
\mathbf{P}^{(2)}=\frac{3(\dot{\mathbf{k}} \cdot \mathbf{k}) \dot{\mathbf{k}}-\omega^{2} \ddot{\mathbf{k}}}{4 \omega^{5}}+\mathcal{N}_{2} \mathbf{k},
$$

where $\mathcal{N}_{2}$ is to be fixed by the normalization requirement. From the expansion (C6), we find to second order

$$
P^{2}=1+\epsilon^{2}\left[P^{(1) 2}+2 \mathbf{P}^{(0)} \cdot \mathbf{P}^{(2)}\right]+\cdots=1 .
$$

So we want to choose $\mathcal{N}_{2}$ such that $P^{(1) 2}+2 \mathbf{P}^{(0)} \cdot \mathbf{P}^{(2)}=$ 0 . This gives the equation

$$
\begin{aligned}
\mathbf{P}^{(0)} \cdot \mathbf{P}^{(2)} & =\frac{3(\dot{\mathbf{k}} \cdot \mathbf{k})^{2}-\omega^{2} \ddot{\mathbf{k}} \cdot \mathbf{k}}{4 \omega^{6}}+\mathcal{N}_{2} \omega \\
& =-\frac{1}{2} P^{(1) 2}=-\frac{|\dot{\mathbf{k}} \times \mathbf{k}|^{2}}{8 \omega^{6}}
\end{aligned}
$$

from which we find

$$
\begin{aligned}
\mathcal{N}_{2} & =-\frac{3(\dot{\mathbf{k}} \cdot \mathbf{k})^{2}-\omega^{2} \ddot{\mathbf{k}} \cdot \mathbf{k}}{4 \omega^{7}}-\frac{|\dot{\mathbf{k}}|^{2} \omega^{2}-(\mathbf{k} \cdot \dot{\mathbf{k}})^{2}}{8 \omega^{7}} \\
& =-\frac{1}{8} \frac{\dot{\pi}_{\eta}^{2}}{\omega^{5}}+\frac{1}{4} \frac{\pi_{\eta} \ddot{\pi}_{\eta}}{\omega^{5}}-\frac{5}{8} \frac{\pi_{\eta}^{2} \dot{\pi}_{\eta}^{2}}{\omega^{7}}
\end{aligned}
$$

So to second adiabatic order, the polarization vector is given by

$$
\begin{aligned}
\mathbf{P} & =\mathbf{P}^{(0)}+\epsilon \mathbf{P}^{(1)}+\epsilon^{2} \mathbf{P}^{(2)}+\cdots \\
& =\frac{\mathbf{k}}{\omega}+\epsilon \frac{\dot{\mathbf{k}} \times \mathbf{k}}{2 \omega^{3}}+\epsilon^{2}\left[\frac{3(\dot{\mathbf{k}} \cdot \mathbf{k}) \dot{\mathbf{k}}-\omega^{2} \ddot{\mathbf{k}}}{4 \omega^{5}}+\mathcal{N}_{2} \mathbf{k}\right]+\cdots
\end{aligned}
$$

In component form, we find

$$
\begin{aligned}
P_{1}= & \frac{\pi_{\eta}}{\omega}-\epsilon^{2}\left(k_{\perp}^{2}+M^{2}\right)\left(\frac{1}{4} \frac{\ddot{\pi}_{\eta}}{\omega^{5}}-\frac{5}{8} \frac{\pi_{\eta} \dot{\pi}_{\eta}^{2}}{\omega^{7}}\right)+\cdots, \\
P_{2}= & \frac{h k_{\perp}}{\omega}-\epsilon M \frac{\dot{\pi}_{\eta}}{2 \omega^{3}}, \quad(\mathrm{C} 21 \mathrm{a}) \\
& +\epsilon^{2} h k_{\perp}\left(-\frac{1}{8} \frac{\dot{\pi}_{\eta}^{2}}{\omega^{5}}+\frac{1}{4} \frac{\pi_{\eta} \ddot{\pi}_{\eta}}{\omega^{5}}-\frac{5}{8} \frac{\pi_{\eta}^{2} \dot{\pi}_{\eta}^{2}}{\omega^{7}}\right)+\cdots, \\
P_{3}= & \frac{M}{\omega}+\epsilon h k_{\perp} \frac{\dot{\pi}_{\eta}}{2 \omega^{3}} \\
& +\epsilon^{2} M\left(-\frac{1}{8} \frac{\dot{\pi}_{\eta}^{2}}{\omega^{5}}+\frac{1}{4} \frac{\pi_{\eta} \ddot{\pi}_{\eta}}{\omega^{5}}-\frac{5}{8} \frac{\pi_{\eta}^{2} \dot{\pi}_{\eta}^{2}}{\omega^{7}}\right)+\cdots,
\end{aligned}
$$

which completes the adiabatic analysis used in this paper. 
[1] T. S. Biró, H. B. Nielson, and J. Knoll, Nuc. Phys. B245, 449 (1984).

[2] F. Sauter, Z. Physik 69, 742 (1931).

[3] W. Heisenberg and H. Euler, Zeit. für Physik 98, 714 (1936).

[4] J. Schwinger, Phys. Rev. 82, 664 (1951).

[5] B. Andersson, G. Gustafson, G. Ingelman, and T. Sjostrand, Phys. Rept. 97, 31 (1983).

[6] X.-N. Wang and M. Gyulassy, Phys. Rev. D 44, 3501 (1991).

[7] L. McLerran and R. Venugopalan, Phys. Rev. D 49, 2233 (1994).

[8] L. McLerran and R. Venugopalan, Phys. Rev. D 49, 3352 (1994).

[9] D. Kharzeev, E. Levin, and K. Tuchin, Phys. Rev. C 75, 044903 (2007).

[10] A. Bialas and W. Czyżn, Phys. Rev. D 30, 2371 (1984).

[11] A. Bialas, W. Czyżn, A. Dyrek, and W. Florkowski, Nuc. Phys. B296, 611 (1988).

[12] K. Kajantie and T. Matsui, Phys. Lett. 164B, 373 (1985).

[13] G. Gatoff, A. K. Kerman, and T. Matsui, Phys. Rev. D 36, 114 (1987).

[14] V. V. Skokov and P. Levai, Phys. Rev. D 78, 054004 (2008).

[15] G. C. Nayak and P. van Nieuwenhuizen, Phys. Rev. D 71, 125001 (2005).

[16] G. C. Nayak, Phys. Rev. D 72, 125010 (2005).

[17] M. Claudson, A. Yildiz, and P. H. Cox, Phys. Rev. D 22, 2022 (1980).

[18] A. Casher, H. Neuberger, and S. Nussinov, Phys. Rev. D
20, 179 (1979).

[19] G. C. Nayak, Phys. Rev. D 72, 125010 (2005).

[20] F. Cooper and E. Mottola, Phys. Rev. D 40, 456 (1989).

[21] Y. Kluger, J. M. Eisenberg, B. Svetitsky, F. Cooper, and E. Mottola, Phys. Rev. Lett. 67, 2427 (1991).

[22] Y. Kluger, J. M. Eisenberg, B. Svetitsky, F. Cooper, and E. Mottola, Phys. Rev. D 45, 4659 (1992).

[23] F. Cooper, J. M. Eisenberg, Y. Kluger, E. Mottola, and B. Svetitsky, Phys. Rev. D 48, 190 (1993).

[24] B. Mihaila, J. F. Dawson, and F. Cooper, Phys. Rev. D 78, 116017 (2008).

[25] F. Cooper, S. Habib, Y. Kluger, E. Mottola, J. P. Paz, and P. R. Anderson, Phys. Rev. D 50, 2848 (1994).

[26] B. Mihaila, J. F. Dawson, and F. Cooper, Phys. Rev. D 74, 036006 (2006).

[27] F. Cooper, G. Frye, and E. Schonberg, Phys. Rev. D 11, 192 (1975).

[28] E. A. Calzetta and B.-L. Hu, Nonequilibrium quantum field theory (Cambridge University Press, Cambridge, 2008).

[29] L. D. Landau, Izv. Akad. Nauk SSSR 17, 51 (1953).

[30] Animations of the proper-time evolution of the longitudinal and transverse momentum-dependent particle-density distributions can be found at http://einstein.unh.edu/BogdanMihaila/qed-3+1/.

[31] B. Mihaila and I. Mihaila, J. Phys. A: Math. Gen. 35, 731 (2002).

[32] J. F. Dawson, B. Mihaila, and F. Cooper, in preparation (2009). 